\title{
New Coins from Old, Smoothly
}

\author{
Olga Holtz $\cdot$ Fedor Nazarov $•$ Yuval Peres
}

Received: 7 April 2009 / Revised: 20 January 2010 / Accepted: 25 January 2010 /

Published online: 11 June 2010

(C) The Author(s) 2010. This article is published with open access at Springerlink.com

\begin{abstract}
Given a (known) function $f:[0,1] \rightarrow(0,1)$, we consider the problem of simulating a coin with probability of heads $f(p)$ by tossing a coin with unknown heads probability $p$, as well as a fair coin, $N$ times each, where $N$ may be random. The work of Keane and O'Brien (ACM Trans. Model. Comput. Simul. 4(2):213$219,1994)$ implies that such a simulation scheme with the probability $\mathbb{P}_{p}(N<\infty)$ equal to 1 exists if and only if $f$ is continuous. Nacu and Peres (Ann. Appl. Probab. 15(1A):93-115, 2005) proved that $f$ is real analytic in an open set $S \subset(0,1)$ if and only if such a simulation scheme exists with the probability $\mathbb{P}_{p}(N>n)$ decaying exponentially in $n$ for every $p \in S$. We prove that for $\alpha>0$ noninteger, $f$ is in the space $C^{\alpha}[0,1]$ if and only if a simulation scheme as above exists with $\mathbb{P}_{p}(N>$ $n) \leq C\left(\Delta_{n}(p)\right)^{\alpha}$, where $\Delta_{n}(x):=\max \{\sqrt{x(1-x) / n}, 1 / n\}$. The key to the proof is
\end{abstract}

Communicated by Carl de Boor.

Research of O. Holtz was supported in part by a Center of Pure and Applied Mathematics grant at UC Berkeley and by the Sofja Kovalevskaja Research Prize of the Humboldt Foundation, Germany. Research of F. Nazarov was supported in part by NSF grants DMS-0501067 and DMS-0800243. Research of Y. Peres was supported in part by NSF grant DMS-0605166.

O. Holtz $(\bowtie)$

Department of Mathematics, University of California-Berkeley, 951 Evans Hall \#3840, Berkeley, CA 94720, USA

e-mail: oholtz@EECS.Berkeley.EDU

O. Holtz

Department of Mathematics, Technische Universität Berlin, MA 4-5, Strasse des 17. Juni 136, Berlin 10623, Germany

F. Nazarov

Department of Mathematics, University of Wisconsin-Madison, 480 Lincoln Drive, Madison, WI 53709, USA

Y. Peres

Theory Group, Microsoft Research, One Microsoft Way, Redmond, WA 98052, USA 
a new result in approximation theory: Let $\mathcal{B}_{n}^{+}$be the cone of univariate polynomials with nonnegative Bernstein coefficients of degree $n$. We show that a function $f$ : $[0,1] \rightarrow(0,1)$ is in $C^{\alpha}[0,1]$ if and only if $f$ has a series representation $\sum_{n=1}^{\infty} F_{n}$ with $F_{n} \in \mathcal{B}_{n}^{+}$and $\sum_{k>n} F_{k}(x) \leq C\left(\Delta_{n}(x)\right)^{\alpha}$ for all $x \in[0,1]$ and $n \geq 1$. We also provide a counterexample to a theorem stated without proof by Lorentz (Math. Ann. 151:239 $251,1963)$, who claimed that if some $\varphi_{n} \in \mathcal{B}_{n}^{+}$satisfy $\left|f(x)-\varphi_{n}(x)\right| \leq C\left(\Delta_{n}(x)\right)^{\alpha}$ for all $x \in[0,1]$ and $n \geq 1$, then $f \in C^{\alpha}[0,1]$.

Keywords Simulation - Approximation order · Positive approximation - Bernstein operator · Lorentz operators · Polynomial reproduction · Smoothness · Hölder class

Mathematics Subject Classification (2000) 41A10 • 41A25 - 65C50 - 41A17 . $68 \mathrm{U} 20 \cdot 41 \mathrm{~A} 35 \cdot 41 \mathrm{~A} 27$

\section{Introduction}

Given a coin with unknown probability of heads $p \in[0,1]$, as well as a fair coin, we would like to simulate a coin with probability of heads $f(p)$ where $f:[0,1] \rightarrow(0,1)$ is a known function. This means that we are allowed to toss the original $p$-coin and the fair coin $N$ times each, where $N$ is an almost surely finite stopping time (a notion to be clarified momentarily) and declare heads or tails, depending on the outcome of these $2 N$ independent coin tosses. The probability of declaring a head must be exactly $f(p)$.

The measure corresponding to tosses of the $p$-coin is the infinite product measure $\mathbb{P}_{p}$ on $\Omega=\{0,1\}^{\mathbb{N}}$, where in each coordinate the weights $(1-p, p)$ are used. A measurable function $N: \Omega \rightarrow \mathbb{N} \cup\{\infty\}$ is a stopping time if for every $k \in \mathbb{N}$, the indicator of $N=k$ is a function of the first $k$ coordinates in $\Omega$. We say that $N$ is almost surely finite if the probability $\mathbb{P}_{p}(N<\infty)$ is 1 . More details on these notions can be found in any graduate textbook in Probability Theory, e.g., [10].

This type of problem goes back to von Neumann's article [9], where he showed how to simulate a fair coin (i.e., $f(p)=1 / 2)$ using only a biased $p$-coin where $p \in(0,1)$. Moreover, the number of tosses $N$ needed satisfies $\mathbb{P}_{p}(N>n) \leq(1-$ $2 \epsilon(1-\epsilon))^{\lfloor n / 2\rfloor}$ if $p \in[\epsilon, 1-\epsilon]$. In this paper we include a fair coin in the simulations since we want to consider $p$ near the endpoints $\{0,1\}$ where simulating a fair coin using a $p$ coin would be slow.

Since von Neumann's article, the simulation problem was subsequently solved for various other classes of functions-see [2, 5-7]. In particular, it was shown in [2] that an $f(p)$-coin can be simulated using finitely many tosses of a $p$-coin for all $p$ in a closed interval $D \subseteq(0,1)$ if and only if $f$ is continuous in $D$. In [5], it was shown that for $f:[0,1] \rightarrow(0,1)$, and $f(p)$-coin can be simulated using finitely many tosses of a $p$-coin via a finite automaton for all $p \in(0,1)$, if and only if $f$ is a rational function over $\mathbb{Q}$. (Simulation via a finite automaton is explained in detail in [5]. An automaton is determined by a finite state space, a finite input alphabet, a transition rule from current state and input symbol to the next state, and a subset of final states. In our context, there are two final states, denoted 0 and 1 , and we require that when the automation is given independent tosses of a $p$-coin as input, it will reach a final state with probability one, and output 1 with probability $f(p)$.) 
In [6], it was shown that if $D \subset(0,1)$ is closed and $f$ is real-analytic in an open neighborhood of $D$, then there is a simulation of an $f(p)$-coin using $N$ tosses of a $p$ coin where $N$ has uniform exponential tails for $p \in D$, and conversely, if a simulation with exponential tails exists for $p$ in an open set $S \subset(0,1)$, then $f$ is real analytic in $S$. Moreover, the problem of simulation was recast in [6] as an approximation problem, and the question of characterizing simulation rates for nonanalytic functions was posed.

Definition 1 Given a simulation algorithm, its simulation rate is the probability $\mathbb{P}_{p}(N>n)$ that the number of required inputs exceeds $n$. (Each input is a toss of a $p$ coin and a toss of a fair coin.) If a simulation algorithm with $\mathbb{P}_{p}(N>n)=O\left(\psi_{n}(p)\right)$ exists, we say that the function $f$ can be simulated at the rate $\psi_{n}(p)$.

The goal of this paper is to show that the simulation rate is determined by the smoothness of the simulated function $f$. Our main result is that for positive $\alpha \notin \mathbb{N}$, a function $f:[0,1] \rightarrow(0,1)$ is in the space $C^{\alpha}$ (defined by a Hölder condition of order $\alpha-r$ on the derivative of order $r:=\lfloor\alpha\rfloor)$ if and only if $f$ can be simulated at the rate $\Delta_{n}(p)^{\alpha}$, where $\Delta_{n}(x):=\max \{\sqrt{x(1-x) / n}, 1 / n\}$, see Theorem 8 below.

\section{Preliminaries and Statement of Results}

We first recall relevant definitions and results from the literature on this problem and from approximation theory. Recall that the univariate Bernstein polynomials of degree $n$ (see, e.g., [4]) are defined as

$$
x \mapsto p_{n k}(x):=\left(\begin{array}{l}
n \\
k
\end{array}\right) x^{k}(1-x)^{n-k}, \quad k=0, \ldots, n .
$$

The Bernstein polynomials of degree $n$ form a basis for the space $\Pi_{n}$ of all polynomials of degree at most $n$. Thus, any polynomial $q$ of degree at most $n$ can be written as

$$
q(x)=\sum_{k=0}^{n} a_{k} p_{n, k}(x),
$$

with the sequence $\left(a_{0}, \ldots, a_{n}\right)$ the degree $n$ Bernstein coefficients of $q$. Whenever we write

$$
q \in \mathcal{B}_{n},
$$

this indicates that $q$ is already represented as a linear combination of the Bernstein polynomials of degree $n$; this is admittedly an abuse of notation since the meaning of " $q \in \mathcal{B}_{n}$ " differs from that of " $q \in \Pi_{n}$ ". In addition, we write

$$
q \in \mathcal{B}_{n}^{+}
$$

whenever the degree $n$ Bernstein coefficients $\left(a_{0}, \ldots, a_{n}\right)$ of $q$ are nonnegative. We will also need the following partial order on the space $\Pi_{n}$ : 
Definition 2 Given $q, r \in \Pi_{n}$, we write $q \preceq_{n} r$, or $r \succeq_{n} q$, to denote that $r-q \in \mathcal{B}_{n}^{+}$.

Result 3 below was established in [6] using a simple probabilistic construction. This result reduces the original simulation question to a problem in approximation theory, which we address in this paper. In [6] the goal was to obtain a simulation for $p$ in a closed subset of $(0,1)$; in this case a fair coin is not needed, as it can be produced from the $p$-coin using the von Neumann algorithm. In the present paper we allow $p$ to range in the whole interval $[0,1]$, so we use a fair coin in addition to the unknown $p$-coin.

Result 3 [6] If there exists an algorithm that simulates a function $f$ on a set $D \subset$ $[0,1]$ using a random finite number $N$ of tosses of a $p$-coin, then for all $n \geq 1$ there exist univariate polynomials

$$
\begin{aligned}
& g_{n}(x):=\sum_{k=0}^{n}\left(\begin{array}{l}
n \\
k
\end{array}\right) a(n, k) x^{k}(1-x)^{n-k}, \\
& h_{n}(x):=\sum_{k=0}^{n}\left(\begin{array}{l}
n \\
k
\end{array}\right) b(n, k) x^{k}(1-x)^{n-k}
\end{aligned}
$$

with the following properties:

(i) $0 \leq a(n, k) \leq b(n, k) \leq 1$;

(ii) $\left(\begin{array}{l}n \\ k\end{array}\right) a(n, k)$ and $\left(\begin{array}{l}n \\ k\end{array}\right) b(n, k)$ are integers;

(iii) $g_{n}(p) \leq f(p) \leq h_{n}(p)$;

(iv) for all $m<n$ we have $g_{m} \preceq_{n} g_{n}$ and $h_{m} \succeq_{n} h_{n}$;

(v) $h_{n}(p)-g_{n}(p)=\mathbb{P}_{p}(N>n)$.

Conversely, if there exist polynomials $g_{n}, h_{n}$ as in (2) satisfying (i)-(iv) with $\lim _{n} h_{n}(p)-g_{n}(p)=0$ for all $p \in D$, then there exists an algorithm that simulates an $f(p)$-coin for all $p \in D$ using $N$ tosses of the $p$-coin, where the random time $N$ satisfies

$$
\mathbb{P}_{p}(N>n)=h_{n}(p)-g_{n}(p) .
$$

As noted in [6], given polynomials $g_{n}, h_{n}$ that satisfy all the requirements except (ii), one can always round the values $\left(\begin{array}{l}n \\ k\end{array}\right) a(n, k)$ down and the values $\left(\begin{array}{l}n \\ k\end{array}\right) b(n, k)$ up to an integer, and the resulting increase in the gap $h_{n}(p)-g_{n}(p)$ is exponentially small in $n$ provided that $p \in[\epsilon, 1-\epsilon]$ for some $\epsilon>0$. In the setting of the present paper, when the $p$-coin is tossed $n$ times we also toss a fair coin $n$ times; this means that condition (ii) above is replaced by

(ii') $\left(\begin{array}{l}n \\ k\end{array}\right) a(n, k)$ and $\left(\begin{array}{l}n \\ k\end{array}\right) b(n, k)$ are integer multiples of $2^{-n}$,

since the probabilities of events that can be generated by tossing a fair coin $n$ times are precisely the integer multiples of $2^{-n}$. Thus, given polynomials $g_{n}, h_{n}$ that satisfy requirements (i), (iii), and (iv), we can round the values $\left(\begin{array}{l}n \\ k\end{array}\right) a(n, k)$ down and the values $\left(\begin{array}{l}n \\ k\end{array}\right) b(n, k)$ up to the nearest multiple of $2^{-n}$; this will only add at most $2^{1-n}$ to the gap $h_{n}-g_{n}$. 
Therefore (up to an additional error term of $2^{1-n}$ ), the problem of determining the rate of simulation in our setting is equivalent to the problem of determining the order of two-sided approximation to $f$, by polynomials $g_{n}, h_{n} \in \mathcal{B}_{n}$ that satisfy requirements (i), (iii), and (iv). We will refer to requirements (iv) as the consistency requirements, to the approximation scheme $\left(g_{n}\right)$ as a Bernstein-positive consistent approximation from below, and to the approximation scheme $\left(h_{n}\right)$ as a Bernsteinpositive consistent approximation from above.

Observe that a Bernstein-positive consistent approximation to a function $f$ from below is equivalent to a certain nonnegative series representation of $f$. Here is a precise statement:

Lemma 4 Let $D \subset[0,1]$, and let $\left(\psi_{n}\right)$ be a nonincreasing sequence of positive functions on $D$ that converges uniformly to 0 . A function $f$ is approximable on $D$ by $a$ sequence of Bernstein-nonnegative polynomials $\left(g_{n}\right)$ of degree $n$ satisfying the consistency requirement (iv)

$$
g_{m} \preceq_{n} g_{n} \text { for all } n \geq m
$$

and the estimate

$$
0 \leq f(x)-g_{n}(x) \leq \psi_{n}(x) \quad \text { for all } x \in D
$$

if and only if $f$ can be represented as a series

$$
f(x)=\sum_{n=0}^{\infty} F_{n}(x), \quad \text { where } \sum_{n>N} F_{n}(x) \leq \psi_{N}(x) \quad \text { for all } x \in D,
$$

and where each $F_{n}$ is a polynomial in Bernstein form of degree $n$ with nonnegative coefficients.

Proof Given an approximation scheme $\left(g_{n}\right)$ as above, set $F_{n}(x):=g_{n}(x)-g_{n-1}(x)$ where the second term $g_{n-1}(x)$ is rewritten in Bernstein form of degree $n$ and where $g_{0}(x):=0$. The consistency requirement (3) then guarantees that the Bernstein coefficients of $F_{n}$ are nonnegative, and the sum $\sum_{n>N} F_{n}(x)$ telescopes into $f(x)-g_{N}(x)$, which is bounded pointwise by $\psi_{N}(x)$ according to (4).

Conversely, given a series representation (5), let $g_{n}(x):=\sum_{k \leq n} F_{k}(x)$. Since the difference $F_{n}(x):=g_{n}(x)-g_{n-1}(x)$ is a Bernstein polynomial with nonnegative coefficients, the polynomials $\left(g_{n}\right)$ satisfy the consistency requirement (3). Also, $f(x)-g_{n}(x)=\sum_{k>n} F_{k}(x) \leq \psi_{n}(x)$ due to the rate condition (4).

This approximation problem can be contrasted with the classical approximation of a given function by (unrestricted) polynomials of degree at most $n$ on the interval $[0,1]$. In that case, the approximation order coincides with the smoothness of $f$. To state this classical result precisely, we first recall how smoothness is measured.

Definition 5 Let $\alpha>0$ with $\alpha \notin \mathbb{N}$. A function $f$ is said to be in the smoothness class $C^{\alpha}[0,1]$ if $f$ is $r:=\lfloor\alpha\rfloor$ times differentiable and the following condition holds: 
The modulus of continuity of the $r$ th derivative $f^{(r)}$

$$
\omega\left(f^{(r)} ; h\right):=\sup _{x, y \in[0,1],|x-y|<h}\left|f^{(r)}(x)-f^{(r)}(y)\right|
$$

is of order $O\left(h^{\alpha-r}\right)$. In that case, we will use the notation

$$
\|f\|_{C^{\alpha}}:=\sup _{h>0} \frac{\omega\left(f^{(r)} ; h\right)}{h^{\alpha-r}} .
$$

(Note that this is a seminorm rather than a norm, as it vanishes on polynomials of degree at most $r$.)

The order of approximation of a given function by polynomials is then determined as follows.

Result 6 (see, e.g., [1, Chap. 8, Theorem 6.3]) Let $\alpha>0$ be a noninteger. There exists a sequence of polynomials $\left\{p_{n}\right\}$, where the degree of $p_{n}$ is at most $n$, satisfying

$$
\left|p_{n}(x)-f(x)\right|=O\left(\left(\Delta_{n}(x)\right)^{2 \alpha}\right) \quad \text { for all } x \in[0,1],
$$

if and only if $f \in C^{\alpha}[0,1]$. Here the quantity $\Delta_{n}(x)$ is defined by

$$
\Delta_{n}:=\Delta_{n}(x):=\max \left\{\sqrt{\frac{x(1-x)}{n}}, \frac{1}{n}\right\} .
$$

In other words, the rate of approximation of $f \in C^{\alpha}[0,1]$ is $O\left(n^{-\alpha}\right)$ away from the boundary of the interval $[0,1]$ and is $O\left(n^{-2 \alpha}\right)$ close to the endpoints 0 and 1 . The characterization of the rate of polynomial approximation for integer values $\alpha$ involves the generalized Zygmund class, which we will recall in Sect. 7. In the main part of this paper, we work under the assumption $\alpha \notin \mathbb{N}$.

Result 6 shows that a function $f \in C^{\alpha}[0,1] \backslash C^{\alpha+\epsilon}[0,1]$ cannot be simulated at the rate $O\left(\Delta_{n}^{2(\alpha+\epsilon)}\right)$. However, since our approximants must satisfy special restrictions imposed by Result 3, we should not expect to achieve the approximation order provided by unrestricted polynomials of degree $n$.

In view of requirement (i), it is natural to consider first the approximation order achieved by polynomials with nonnegative Bernstein coefficients. G.G. Lorentz proposed a solution to this problem in [3], where he argued that the approximation order under this constraint is half the approximation order provided by unconstrained polynomials, i.e., half the smoothness of the function $f$. Theorem 1 of [3] establishes that a $C^{\alpha}$-function $f$ can be approximated at the rate $O\left(\left(\Delta_{n}\right)^{\alpha}\right)$ by Bernstein-nonnegative polynomials of degree $n$.

Result 7 [3, Theorem 1] Let $\alpha>0$. A positive function $f \in C^{\alpha}[0,1]$ can be approximated by polynomials $q_{n}$ of degree at most $n$ with nonnegative Bernstein coefficients at the rate

$$
\left|q_{n}(x)-f(x)\right|=O\left(\left(\Delta_{n}(x)\right)^{\alpha}\right) \quad \text { for all } x \in[0,1] .
$$


Lorentz [3] also stated (without proof) a converse to this result; unfortunately, that converse is incorrect. We return to this point at the end of the section.

We will use a variant of Lorentz' approach to establish our main result, that with the extra requirements (i), (iii), and (iv) in place, we can still achieve the same approximation order as in (6).

Theorem 8 Let $f:[0,1] \rightarrow(0,1)$, and let $\alpha>0$ with $\alpha \notin \mathbb{N}$. If $f \in C^{\alpha}[0,1]$, then $f$ can be simulated at the rate $\left(\Delta_{n}(x)\right)^{\alpha}$ on $[0,1]$. Precisely, there exist polynomials $g_{n}$ and $h_{n}$ satisfying conditions (i), (ii'), (iii), and (iv) of Result 3 and $h_{n}(x)-g_{n}(x)=O\left(\left(\Delta_{n}(x)\right)^{\alpha}\right)$ uniformly in $[0,1]$. Conversely, if $f$ can be simulated at the rate $\left(\Delta_{n}(x)\right)^{\alpha}$ on the interval $[0,1]$, then $f \in C^{\alpha}[0,1]$.

We begin by proving a reduction lemma that shows that it is enough to find consistent approximants $g_{n}, h_{n}$ for each $b$-adic degree $n \in b^{\mathbb{N}}=\left\{b^{\ell}\right\}_{\ell \geq 1}$ where $b$ is a fixed integer greater than 1 . Using these approximants, one can then interpolate between $b$-adic levels to build up a consistent approximation scheme providing the same approximation order as the $b$-adic polynomials $g_{n}, h_{n}, n=b^{\ell}$. This $b$-adic idea per se is quite well known and, in particular, is used in [6] with $b=2$.

Lemma 9 Let $b$ be a fixed integer greater than 1. Given a function $f$ on $[0,1]$, suppose there exist two sequences of polynomials $\left(g_{n}\right)_{n \in b^{\mathbb{N}}},\left(h_{n}\right)_{n \in b^{\mathbb{N}}}$ satisfying conditions (i), (iii), and (iv) of Result 3 with $\psi_{n}$ of order $O\left(\left(\Delta_{n}\right)^{\alpha}\right)$, so that

$$
h_{n}(x)-g_{n}(x)=O\left(\left(\Delta_{n}(x)\right)^{\alpha}\right) \quad \text { uniformly in }[0,1]
$$

for $n \in b^{\mathbb{N}}$. Then these sequences can be augmented to full sequences $\left(g_{n}\right)_{n \in \mathbb{Z}_{+}}$, $\left(h_{n}\right)_{n \in \mathbb{Z}_{+}}$satisfying conditions (i), (iii), and (iv) from Result 3 and condition (7) for all $n \in Z_{+}$. In particular, under these assumptions there exists an algorithm that simulates an $f(p)$-coin at the rate $O\left(\left(\Delta_{n}(p)\right)^{\alpha}\right)$ on $[0,1]$.

Proof Given the polynomials $g_{n}$ and $h_{n}$ for $b$-adic values of $n$, we will fill in the gaps in the two sequences in the obvious way: given $n$, let $n^{\prime}:=b^{\left\lfloor\log _{b} n\right\rfloor}$, and set

$$
g_{n}(x):=(x+(1-x))^{n-n^{\prime}} g_{n^{\prime}}(x), \quad h_{n}(x):=(x+(1-x))^{n-n^{\prime}} h_{n^{\prime}}(x)
$$

by expanding the right-hand sides into Bernstein polynomials of degree $n$. The Bernstein coefficients of the resulting polynomials $g_{n}, h_{n}$ are therefore some convex combinations of the coefficients of $g_{n^{\prime}}, h_{n^{\prime}}$. It follows that condition (i) holds for the full sequences $\left(g_{n}\right),\left(h_{n}\right)$. It is clear from the construction that (iii) and (iv) hold as well, the latter condition being an equality except when jumping from one $b$-adic level to the next, when it is satisfied by our assumption. Recall that condition (ii') can always be satisfied by introducing an exponentially small correction, so there is no need to verify it explicitly. To check that (7) holds for the full sequences $\left(g_{n}\right),\left(h_{n}\right)$, note that, by construction,

$$
h_{n}(x)-g_{n}(x)=h_{n^{\prime}}(x)-g_{n^{\prime}}(x)=O\left(\left(\Delta_{n^{\prime}}(x)\right)^{\alpha}\right) .
$$


But since $n^{\prime} \leq n<b n^{\prime}$, we see that $O\left(\left(\Delta_{n^{\prime}}(x)\right)^{\alpha}\right)=O\left(\left(\Delta_{n}(x)\right)^{\alpha}\right)$. This completes the proof.

As noted already, Lorentz [3] stated a converse to Result 7 above, which (in a special case) can be written as follows:

Claim 10 [3, Theorems 5 and 6] Let $\alpha>0$. If a function $f$ can be approximated by polynomials $q_{n}$ of degree at most $n$ with nonnegative Bernstein coefficients at the rate (6), then $f \in C^{\alpha}[0,1]$.

The argument proposed in [3] for these theorems skips technical details and refers to the work of Timan [8]. Specifically, we quote Theorem 6 from [3] and the subsequent discussion:

" Theorem 6. For each $r=1,2, \ldots$ there is a constant $C_{r}$ with the following property. Let $\omega(h)$ be a modulus of continuity, and put

$$
\tilde{\omega}(h)=h \int_{h}^{1} \frac{\omega(u)}{u^{2}} \mathrm{~d} u+\int_{0}^{h} \frac{\omega(u)}{u} \mathrm{~d} u .
$$

If $f(x)$ is a continuous function on $[0,1]$ and if there exists a sequence $P_{n}(x)$ of polynomials with positive coefficients of degree $n$ such that

$$
\left|f(x)-P_{n}(x)\right| \leq\left(\Delta_{n}\right)^{r} \omega\left(\Delta_{n}\right), \quad 0 \leq x \leq 1, n=0,1, \ldots,
$$

then $f$ has on $[0,1]$ the continuous derivatives $f^{\prime}, f^{\prime \prime}, \ldots, f^{(r)}$ and

$$
\omega\left(f^{(r)} ; h\right) \leq C_{r} \tilde{\omega}(h) .
$$

We omit the proofs. The method of deriving theorems of this kind from inequalities of the Markov-Bernstein type is due essentially to S. Bernstein, and is well known. For the variation of it which fits the present situation especially well, compare [6, p. 357 and p. 360]. It should be noted that [6, p. 357] contains an essential mistake: the derivative $P_{2^{m+1}}^{\prime}$ on $\mathrm{p} .359$ should have been estimated at a point different from $x$. However, the proof can be corrected."

In this quote, [6] refers to the original Russian edition of Timan's work [8]. Trying to reconstruct Lorentz' complete argument for his Theorem 6, we came to the realization that his argument requires an extra assumption, in fact precisely the assumption of Bernstein-nonnegative consistent approximation, or equivalently, the nonnegative series representation (5) that is central to this paper. In the next section we show that, indeed, such a series representation of $f$ with tails decaying at the rate $\left(\Delta_{n}\right)^{\alpha}$ implies the $C^{\alpha}$ smoothness of the represented function $f$. Thus our results here also provide a correction to the statement of Lorentz. In Sect. 6, we construct a counterexample to Theorem 6 from [3].

Our final point in this section concerns notation. In the rest of the paper, we will establish a number of estimates on various functions. The constants in such estimates will usually simply be denoted by const or, say, const $_{j}$, the latter indicating that the constant may depend on $j$. A few constants that are crucial to our main argument will be labeled by the number of the theorem or lemma where they occur. 


\section{Consistent Approximation Implies Smoothness in Theorem 8}

Lorentz proved the following analogs of Bernstein's and Markov's inequalities (both original inequalities can be found, e.g., in [1]). This result of Lorentz is formulated for a certain class of functions $\Omega$; we will use it only for the power functions $t \mapsto t^{j}$.

Result 11 [3, Theorem 3] For each $r=1,2, \ldots$ and each $H>0$, there is a constant $K_{r}=K_{r}(H)$ with the following property. If $\Omega(h)$ is an increasing positive function defined for all $h \geq 0$ such that

$$
\Omega(2 h) \leq H \Omega(h), \quad h \geq 0,
$$

then for each Bernstein-positive polynomial $P_{n}$ of degree $n$, the inequality

$$
P_{n}(x) \leq \Omega\left(\Delta_{n}(x)\right), \quad 0 \leq x \leq 1
$$

implies

$$
\left|P_{n}^{(r)}(x)\right| \leq K_{r}\left(\Delta_{n}(x)\right)^{-r} \Omega\left(\Delta_{n}(x)\right), \quad 0 \leq x \leq 1 .
$$

We need the following observation:

Lemma 12 For any $x$ and $\xi$ in $[0,1]$,

$$
\max \left\{\frac{\Delta_{n}(\xi)}{\Delta_{n}(x)}, \frac{\Delta_{n}(x)}{\Delta_{n}(\xi)}\right\} \leq 2\left(1+\frac{|x-\xi|}{\Delta_{n}(x)}\right) .
$$

Proof We start by proving one of the two inequalities, viz.,

$$
\frac{\Delta_{n}(x)}{\Delta_{n}(\xi)} \leq 2\left(1+\frac{|x-\xi|}{\Delta_{n}(x)}\right)
$$

By the symmetry $\Delta_{n}(x)=\Delta_{n}(1-x)$, we may assume that $x, \xi \in[0,1 / 2]$. We also assume that $\xi<x$ and $\Delta_{n}(x)>1 / n$, since otherwise the inequality is obvious. If $\xi \geq x / 2$, then the left-hand side of (9) is at most 2 , so we may assume that $\xi<x / 2$. In this case we have

$$
\left(\Delta_{n}(x)\right)^{2} \leq x / n \leq 2|x-\xi| / n \leq 2|x-\xi| \Delta_{n}(\xi),
$$

which implies (9).

The proof of the other inequality (which bounds $\Delta_{n}(\xi) / \Delta_{n}(x)$ by the right-hand side of (9)) is very similar. We may again assume, by symmetry, that $x, \xi \in[0,1 / 2]$. We also assume that $\xi>x$ and $\Delta_{n}(\xi)>1 / n$ since otherwise the inequality is obvious. Thus $\Delta_{n}(\xi) \leq \sqrt{\xi / n}<\xi$. If $\xi \leq 2 x$, then the left-hand side is at most 2 , while the right-hand side is greater than 2 . Thus, the only remaining case is $\xi>2 x$. Then

$$
\Delta_{n}(\xi) \leq \xi \leq 2|\xi-x|
$$

Dividing by $\Delta_{n}(x)$, we obtain the desired bound. 
To prove the necessity of $C^{\alpha}$-smoothness, we will follow the approach suggested by G. Lorentz in [3], which goes back to Timan [8] and ultimately to S. Bernstein.

Proof of necessity in Theorem 8 Suppose that $f$ can be simulated at the rate $\left(\Delta_{n}\right)^{\alpha}$ on the interval $[0,1]$. Using the sequence $\left(g_{n}\right)$ that approximates $f$ from below and satisfies the consistency requirement $g_{n} \preceq_{2 n} g_{2 n}$, we set $G_{n}:=g_{2^{n+1}}-g_{2^{n}}$ and obtain the following nonnegative series representation for $f$ :

$$
f(x)=\sum_{n=0}^{\infty} G_{n}(x), \quad G_{n} \in \mathcal{B}_{2^{n}}^{+} .
$$

By the assumption on the rate of approximation, the polynomials $G_{n}$ satisfy the bound

$$
\left|G_{n}(x)\right| \leq \operatorname{const}\left(\Delta_{2^{n}}(x)\right)^{\alpha} \quad \text { for all } x \in[0,1] .
$$

Now the inequality (8) implies

$$
\left|G_{n}^{(j)}(x)\right| \leq \operatorname{const}\left(\Delta_{2^{n}}(x)\right)^{\alpha-j} \quad \text { for all } x \in[0,1], j \in \mathbb{N} .
$$

This already ensures that we can differentiate (10) term by term $r$ times, and that

$$
f^{(r)}(x)=\sum_{n=0}^{\infty} G_{n}^{(r)}(x)
$$

is continuous in $[0,1]$. Our goal is to prove that $f \in C^{\alpha}[0,1]$, i.e., that the inequality

$$
\left|f^{(r)}(x)-f^{(r)}(y)\right| \leq \text { const }|x-y|^{\alpha-r}
$$

holds for $x, y \in[0,1]$. Without loss of generality $x(1-x) \geq y(1-y)$, whence $\Delta_{n}(x) \geq \Delta_{n}(y)$ for all $n$. For any $n$, there is some $\xi_{n}$ between $x$ and $y$ such that

$$
\left|G_{n}^{(r)}(x)-G_{n}^{(r)}(y)\right|=|x-y|\left|G_{n}^{(r+1)}\left(\xi_{n}\right)\right| \leq \mathrm{const}|x-y|\left(\Delta_{2^{n}}\left(\xi_{n}\right)\right)^{\alpha-r-1},
$$

using the bound (11) with $j=r+1$. Choose $N$ so that

$$
\Delta_{2^{N+1}}(x)<|x-y| \leq \Delta_{2^{N}}(x) .
$$

For $n \leq N$ we have $|x-y| \leq \Delta_{2^{n}}(x)$, so Lemma 12 implies that $\Delta_{2^{n}}(x) \leq 4 \Delta_{2^{n}}\left(\xi_{n}\right)$. Thus for $n \leq N$, (14) gives

$$
\left|G_{n}^{(r)}(x)-G_{n}^{(r)}(y)\right| \leq \text { const }|x-y|\left(\Delta_{2^{n}}(x)\right)^{\alpha-r-1} .
$$

We now write $f^{(r)}(x)-f^{(r)}(y)$ by splitting the sum (12) into two parts:

$$
f^{(r)}(x)-f^{(r)}(y)=\sum_{n=0}^{N}\left(G_{n}^{(r)}(x)-G_{n}^{(r)}(y)\right)+\sum_{n=N+1}^{\infty}\left(G_{n}^{(r)}(x)-G_{n}^{(r)}(y)\right) .
$$


Estimate the first sum using (16) and the second using (11) with $j=r$. This yields

$$
\begin{aligned}
\left|f^{(r)}(x)-f^{(r)}(y)\right| & \leq \text { const }\left(|x-y| \sum_{n=0}^{N}\left(\Delta_{2^{n}}(x)\right)^{\alpha-r-1}+2 \sum_{n=N+1}^{\infty}\left(\Delta_{2^{n}}(x)\right)^{\alpha-r}\right) \\
& \leq \text { const }|x-y|\left(\Delta_{2^{N}}(x)\right)^{\alpha-r-1}+\operatorname{const}\left(\Delta_{2^{N+1}}(x)\right)^{\alpha-r}
\end{aligned}
$$

where we used the inequality $\Delta_{k}(x) \geq \sqrt{2} \Delta_{2 k}(x)$ to compare the two series in (18) to geometric series. In view of (15), the bound (19) yields (13).

In the preceding proof, the strict inequality $\alpha<r+1$ is used only at one point: to show that the sum of terms with $n \leq N$ in (17) is comparable to the last term. (If $\alpha=r+1$, then all these terms are of the same magnitude and we lose a factor of $N \approx \log \frac{1}{|x-y|}$ in the estimate.) Nevertheless, for the case $\alpha=r+1$, the same method will allow us to show that $f^{(r)}$ is in the Zygmund class.

Theorem 13 Let $r$ be a nonnegative integer. Suppose that $f:[0,1] \rightarrow(0,1)$ can be simulated at the rate $\left(\Delta_{n}(x)\right)^{r+1}$ on $[0,1]$. Precisely, suppose that there exist polynomials $g_{n}$ and $h_{n}$ satisfying conditions (i), (ii'), (iii), and (iv) of Result 3 and $h_{n}(x)-g_{n}(x)=O\left(\Delta_{n}^{r+1}(x)\right)$ uniformly in $[0,1]$. Then $f^{(r)}$ is in the Zygmund class, that is

$$
\left|f^{(r)}(x+\delta)-2 f^{(r)}(x)+f^{(r)}(x-\delta)\right|=O(\delta),
$$

uniformly for all $x, \delta$ such that $0 \leq x-\delta<x+\delta \leq 1$.

In fact, as in the preceding proof, only the approximation from below by $g_{n}$ is used.

Proof The hypothesis implies that $f$ has a series representation as in (10) where the polynomials $G_{n} \in \mathcal{B}_{2^{n}}^{+}$satisfy $\left|G_{n}(x)\right| \leq \operatorname{const}\left(\Delta_{2^{n}}(x)\right)^{r+1}$ for all $x \in[0,1]$. The inequality (8) implies

$$
\left|G_{n}^{(j)}(x)\right| \leq \operatorname{const}\left(\Delta_{2^{n}}(x)\right)^{r+1-j} \quad \text { for all } x \in[0,1], j \in \mathbb{N},
$$

so (12) holds and $f^{(r)}$ is continuous in [0,1]. Fix $\delta \in(0,1 / 2)$, and choose $N$ minimal so that $\delta \leq \Delta_{2^{N}}(x)$. Write $f=S_{1}+S_{2}$, where

$$
S_{1}=\sum_{n=0}^{N} G_{n}(x) \quad \text { and } \quad S_{2}=\sum_{n=N+1}^{\infty} G_{n}(x) .
$$

The preceding proof works to show that $S_{2}(x+\delta)-S_{2}(x)=O(\delta)$, and this implies that the estimate (20) holds with $S_{2}^{(r)}$ in place of $f^{(r)}$. It remains to handle $S_{1}^{(r)}$. 
For any $n$, there is some $\eta_{n} \in[x-\delta, x+\delta]$ such that

$$
\begin{aligned}
\left|G_{n}^{(r)}(x+\delta)-2 G_{n}^{(r)}(x)+G_{n}^{(r)}(x-\delta)\right| & =\delta^{2}\left|G_{n}^{(r+2)}\left(\eta_{n}\right)\right| \\
& \leq \operatorname{const} \delta^{2}\left(\Delta_{2^{n}}\left(\eta_{n}\right)\right)^{-1}
\end{aligned}
$$

using the bound (21) with $j=r+2$.

For $n \leq N$ we have $\delta \leq \Delta_{2^{n}}(x)$, so Lemma 12 implies that $\Delta_{2^{n}}(x) \leq 4 \Delta_{2^{n}}\left(\eta_{n}\right)$. Thus for $n \leq N$, (22) gives

$$
\left|G_{n}^{(r)}(x+\delta)-2 G_{n}^{(r)}(x)+G_{n}^{(r)}(x-\delta)\right| \leq \operatorname{const} \delta^{2}\left(\Delta_{2^{n}}(x)\right)^{-1} .
$$

This yields

$$
\begin{aligned}
\left|S_{1}^{(r)}(x+\delta)-2 S_{1}^{(r)}(x)+S_{1}^{(r)}(x+\delta)\right| & \leq \operatorname{const}\left(\delta^{2} \sum_{n=0}^{N}\left(\Delta_{2^{n}}(x)\right)^{-1}\right) \\
& \leq \operatorname{const} \delta^{2}\left(\Delta_{2^{N}}(x)\right)^{-1} \leq \operatorname{const} \delta
\end{aligned}
$$

The previous estimate for $S_{2}$, together with the bound (23), yields (20).

\section{Lorentz Operators and Simultaneous Approximation}

In the following three sections, we restrict attention to $\alpha \notin \mathbb{N}$. We now recall the main ingredients of the valid proof of Result 7 (Theorem 1 from [3]). That proof is based on the Taylor expansion

$$
f(x)=f\left(\frac{k}{n}\right)-\sum_{j=1}^{r} \frac{1}{j !}\left(\frac{k}{n}-x\right)^{j} f^{(j)}(x)+\frac{1}{r !}\left(\frac{k}{n}-x\right)^{r}\left[f^{(r)}(x)-f^{(r)}\left(\xi_{k}\right)\right],
$$

where $\xi_{k}:=\xi_{k}(x)$ is a point between $x$ and $k / n$ and $f$ is assumed to be $r$ times differentiable. This formula is used in [3] to derive an asymptotic expansion of the Bernstein operator

$$
\left(B_{n} f\right)(x):=\sum_{k=0}^{n} f\left(\frac{k}{n}\right) p_{n k}(x),
$$

where the polynomials $p_{n k}$ are defined in (1). Multiplying the Taylor expansion (24) by $p_{n k}(x)$ and summing over $k$, we obtain

$$
\begin{aligned}
f(x) & =\left(B_{n} f\right)(x)-\sum_{j=1}^{r} \frac{1}{j ! n^{j}} T_{n j}(x) f^{(j)}(x)+\left(R_{r} f\right)(x), \quad \text { where } \\
T_{n j}(x) & :=\sum_{k=0}^{n}(k-n x)^{j} p_{n k}(x),
\end{aligned}
$$




$$
\left(R_{r} f\right)(x):=\frac{1}{r !} \sum_{k=0}^{n}\left(\frac{k}{n}-x\right)^{r}\left[f^{(r)}(x)-f^{(r)}\left(\xi_{k}\right)\right] p_{n k}(x) .
$$

This leads Lorentz to the natural definition of the operators $Q_{n, r}$, using the recurrence

$$
\left(Q_{n, r} f\right)(x):=\left(B_{n} f\right)(x)-\sum_{j=1}^{r} \frac{1}{j ! n^{j}} T_{n j}(x)\left(Q_{n, r-j} f^{(j)}\right)(x),
$$

where each $f^{(j)}$ in (25) is replaced by its approximation $Q_{n, r-j} f^{(j)}$.

Note that the sum in (27) in fact starts at $j=2$ rather than at $j=1$, since the polynomial $T_{n 1}$ is identically zero. Also note that the expressions (27) must be written in the Bernstein basis of degree $n+r$, so that, e.g., the leading term $B_{n} f$ must be multiplied by the binomial expansion of $(x+(1-x))^{r}$ to appear in its Bernstein form of degree $n+r$. We will refer to the operators $Q_{n, r}$ mapping a function to a polynomial in Bernstein form of degree $n+r$ as the Lorentz operators.

An important property of the Lorentz operators that can be inferred directly from their recursive definition is their polynomial reproduction. Precisely, the Lorentz operator $Q_{n, r}$ reproduces polynomials of degree at most $r$.

Lemma 14 Let $f$ be a polynomial of degree at most $r$. Then $Q_{n, r} f=f$.

Proof The proof is by induction on $r$. The result holds for $r=0$ and 1 since $Q_{n, 0}=$ $Q_{n, 1}$ is simply the Bernstein operator, which reproduces linear functions. For higher values of $r$, the proof is as follows. The Taylor polynomial of $f$ of degree $r$ coincides with $f$, so

$$
f(x)=f\left(\frac{k}{n}\right)-\sum_{j=1}^{r} \frac{1}{j !}\left(\frac{k}{n}-x\right)^{j} f^{(j)}(x),
$$

so by multiplying by $p_{n k}(x)$ and summing over $k$, we obtain

$$
f(x)=\left(B_{n} f\right)(x)-\sum_{j=1}^{r} \frac{1}{j ! n^{j}} T_{n j}(x) f^{(j)}(x) .
$$

By our inductive assumption, $f^{(j)}=Q_{n, r-j} f^{(j)}$. Substituting this into (27), we get $Q_{n, r} f=f$.

As noted in [3], the Lorentz operators can be rewritten as follows

$$
\left(Q_{n, r} f\right)(x)=: \sum_{k=0}^{n}\left(f\left(\frac{k}{n}\right)+\sum_{j=2}^{r} f^{(j)}\left(\frac{k}{n}\right) \frac{1}{n^{j}} \tau_{r j}(x, n)\right) p_{n k}(x),
$$

or, more simply, as

$$
\left(Q_{n, r} f\right)(x)=\sum_{k=0}^{n}\left(\sum_{j=0}^{r} f^{(j)}\left(\frac{k}{n}\right) \frac{1}{n^{j}} \tau_{r j}(x, n)\right) p_{n k}(x),
$$


with the understanding that $\tau_{r 0}(x, n)=1$ and $\tau_{r 1}(x, n)=0$. Plugging (28) into (27), we obtain

$\left(Q_{n, r} f\right)(x)=\sum_{k=0}^{n}\left(f\left(\frac{k}{n}\right)-\sum_{j=2}^{r} f^{(j)}\left(\frac{k}{n}\right) \frac{1}{n^{j}} \sum_{l=2}^{j} \frac{1}{l !} T_{n l}(x, n) \tau_{r-l, j-l}(x, n)\right) p_{n k}(x)$.

By term-by-term comparison, this yields

$$
\tau_{r j}(x, n)=-\sum_{l=2}^{j} \frac{1}{l !} T_{n l}(x) \tau_{r-l, j-l}(x, n) \quad \text { for } j \geq 2 .
$$

The recurrence (29) can be used to show that the polynomials $\tau_{r j}(x, n)$ are independent of $f$, and are of degree $j$ in $x$ and of degree $\lfloor j / 2\rfloor$ in $n$, as noted by Lorentz [3]. The recurrence (29) also shows that, as functions, the $\tau_{r j}$ do not depend on the parameter $r$. However, in the expression for $Q_{n, r}$, each of the $\tau_{r j} \mathrm{~s}$ is written in its Bernstein form of degree $r$ to bring the entire expression $Q_{n, r} f$ into its Bernstein form of degree $n+r$. Since we are mainly concerned with pointwise estimates on the $\tau_{r j} \mathrm{~s}$, we will use the simpler notation $\tau_{j}:=\tau_{r j}$. We will begin with the following estimates on the polynomials $\tau_{j}$ :

Lemma 15 The polynomials $\tau_{j}$ are bounded by

$$
\left|\tau_{j}(x, n)\right| \leq \text { const }_{j} n^{j}\left(\Delta_{n}(x)\right)^{j} \quad \text { for all } x \in[0,1],
$$

where const ${ }_{j}$ depends only on $j$.

Proof We use induction on $j$. For $j=0,1$, (30) is clear. By [3, (17) on p. 242],

$$
\left|T_{n \ell}(x)\right| \leq \text { const }_{\ell} n^{l}\left(\Delta_{n}(x)\right)^{\ell} .
$$

Applying (29) and the induction hypothesis

$$
\forall \ell>0, \quad\left|\tau_{j-\ell}(x, n)\right| \leq \text { const }_{j-\ell} n^{j-\ell}\left(\Delta_{n}(x)\right)^{j-\ell}
$$

gives (30), as required.

Corollary 16 Fix an integer $r \geq 0$. For any $j \leq r$, write

$$
\tau_{j}(x, n)=: \sum_{i=0}^{j} a_{i}(n, j) x^{i}(1-x)^{j-i}
$$

Then for all $i \in[0, j]$, we have $\left|a_{i}(n, j)\right|=\left|a_{j-i}(n, j)\right|$ and $\left|a_{i}(n, j)\right| \leq C_{j}^{\sharp} n^{i}$ for some constants $C_{j}^{\sharp}$. 
Proof The polynomials $T_{n j}$ satisfy $T_{n j}(1-x)=(-1)^{j} T_{n j}(x)$, as is easily seen using the substitution $\tilde{k}=n-k$ in their definition (26). It then follows from the recursion (29) that $\tau_{j}(1-x, n)=(-1)^{j} \tau_{j}(x, n)$ as well, and this implies that $\left|a_{i}(n, j)\right|=$ $\left|a_{j-i}(n, j)\right|$ for all $i$. Next, consider the polynomial $A(x):=\sum_{i=0}^{j} a_{i}(n, j) x^{i}$. Since $\tau_{j}(x, n)=(1-x)^{j} A\left(\frac{x}{1-x}\right)$, Lemma 15 implies that $|A(x)| \leq$ const $_{j}$ for $x \in[0,1 / n]$. Thus $A_{*}(x):=A\left(\frac{x+1}{2 n}\right)$ satisfies $\left|A_{*}(x)\right| \leq$ const $_{j}$ for $x \in[-1,1]$. Markov's inequality $\left\|A_{*}^{(i)}\right\|_{\infty} \leq j^{2 i}\left\|A_{*}\right\|_{\infty}$ (see [1, Chap. 4, Theorem 1.4]) yields

$$
\left|a_{i}(n, j)\right|=(i !)^{-1}\left|A^{(i)}(0)\right|=(i !)^{-1}(2 n)^{i}\left|A_{*}^{(i)}(-1)\right| \leq C_{j}^{\sharp} n^{i} \quad \text { for all } i \leq j .
$$

For our next argument, we will need an additional technical lemma that provides bounds on the derivatives of the functions $p_{n k}$.

Lemma 17 For any integer $\ell \geq 0$ and any $\beta \geq 0$,

$$
\sum_{k=0}^{n}\left|\frac{k}{n}-x\right|^{\beta}\left|p_{n k}^{(\ell)}(x)\right| \leq \operatorname{const}_{\beta, \ell}\left(\Delta_{n}(x)\right)^{\beta-\ell} \quad \text { for all } x \in[0,1] .
$$

Proof The proof is by induction on $\ell$. The proof for $\ell=0$ is due to Lorentz [3, Lemma 1]. Our proof of the bound (31) for $\ell \geq 1$ splits into two cases.

Case 1. $\Delta_{n}(x)=\sqrt{x(1-x) / n}$. In this case we start from the equality

$$
p_{n k}^{\prime}(x)=\frac{k-n x}{x(1-x)} p_{n k}(x)
$$

and deduce by induction on $\ell$ that the $\ell$ th derivative of $p_{n k}$ has the form

$$
p_{n k}^{(\ell)}(x)=\sum_{i, j, v \geq 0}\left\{\frac{n^{i}(k-n x)^{v}}{[x(1-x)]^{j}} \Psi_{\ell i j v}(x) p_{n k}(x): i+j \leq \ell ; i+v \leq j\right\},
$$

where $\Psi_{\ell i j v}(x)$ are polynomials in $x$ with coefficients that do not depend on $n$. For fixed $i, j, v$, we have (using that $x(1-x) \geq \Delta_{n}(x)$ in this case)

$$
\frac{n^{i}|k-n x|^{v}}{[x(1-x)]^{j}}=\left(\Delta_{n}(x)\right)^{-2(i+v)} \frac{\left|\frac{k}{n}-x\right|^{v}}{[x(1-x)]^{j-i-v}} \leq\left(\Delta_{n}(x)\right)^{-(i+v+j)}\left|\frac{k}{n}-x\right|^{v},
$$

whence (using the already established case $\ell=0$ of (31)), we obtain

$$
\begin{aligned}
\sum_{k=0}^{n}\left|\frac{k}{n}-x\right|^{\beta} \frac{n^{i}|k-n x|^{v}}{[x(1-x)]^{j}} p_{n k}(x) & \leq\left(\Delta_{n}(x)\right)^{-(i+v+j)} \sum_{k=0}^{n}\left|\frac{k}{n}-x\right|^{\beta+v} p_{n k}(x) \\
& \leq \operatorname{const}_{\beta, \ell}\left(\Delta_{n}(x)\right)^{\beta-i-j} .
\end{aligned}
$$

The restriction $i+j \leq \ell$ implies that the right-hand side of the last display is at most const $_{\beta, \ell}\left(\Delta_{n}(x)\right)^{\beta-\ell}$. The representation (32) completes the proof in this case. 
Case 2. $\Delta_{n}(x)=1 / n$. In this case we substitute a different expression for $p_{n k}^{\prime}(x)$, precisely

$$
p_{n k}^{\prime}(x)=n p_{n-1, k-1}(x)-n p_{n-1, k}(x),
$$

which yields

$$
p_{n k}^{(\ell)}(x)=n p_{n-1, k-1}^{(\ell-1)}(x)-n p_{n-1, k}^{(\ell-1)}(x) .
$$

This gives the bound

$$
\sum_{k=0}^{n}\left|\frac{k}{n}-x\right|^{\beta}\left|p_{n k}^{(\ell)}(x)\right| \leq \operatorname{const}_{\beta, \ell} n \sum_{k=0}^{n}\left|\frac{k}{n}-x\right|^{\beta}\left(\left|p_{n-1, k-1}^{(\ell-1)}(x)\right|+\left|p_{n-1, k}^{(\ell-1)}(x)\right|\right) .
$$

The general inequality $(a+b)^{\beta} \leq 2^{\beta}\left(a^{\beta}+b^{\beta}\right)$ implies that

$$
\begin{aligned}
& \left|\frac{k}{n}-x\right|^{\beta} \leq 2^{\beta}\left(\left|\frac{k-1}{n-1}-x\right|^{\beta}+\left(\frac{n-k}{n(n-1)}\right)^{\beta}\right) \leq 2^{\beta}\left(\left|\frac{k-1}{n-1}-x\right|^{\beta}+\frac{1}{n^{\beta}}\right) \\
& \quad \text { for } k \geq 1, \\
& \left|\frac{k}{n}-x\right|^{\beta} \leq 2^{\beta}\left(\left|\frac{k}{n-1}-x\right|^{\beta}+\left(\frac{k}{n(n-1)}\right)^{\beta}\right) \leq 2^{\beta}\left(\left|\frac{k}{n-1}-x\right|^{\beta}+\frac{1}{n^{\beta}}\right) \\
& \quad \text { for } k<n .
\end{aligned}
$$

Therefore $\sum_{k=0}^{n}\left|\frac{k}{n}-x\right|^{\beta}\left|p_{n k}^{(\ell)}(x)\right|$ is at most

$$
\begin{aligned}
& 2^{\beta} n \sum_{k=1}^{n}\left(\left|\frac{k-1}{n-1}-x\right|^{\beta}+n^{-\beta}\right)\left|p_{n-1, k-1}^{(\ell-1)}(x)\right| \\
& \quad+2^{\beta} n \sum_{k=0}^{n-1}\left(\left|\frac{k}{n-1}-x\right|^{\beta}+n^{-\beta}\right)\left|p_{n-1, k}^{(\ell-1)}(x)\right| .
\end{aligned}
$$

Since $\frac{n-1}{n} \Delta_{n-1} \leq \Delta_{n} \leq \Delta_{n-1}$, we can finish the proof using the inductive assumption on $\ell-1$.

We now generalize Lemma 15 to derive bounds on the derivatives of the polynomials $\tau_{j}$.

Lemma 18 The derivatives of the polynomials $T_{n j}$ and $\tau_{j}$ are bounded as follows:

$$
\begin{array}{r}
\left|T_{n j}^{(\ell)}(x)\right| \leq \text { const }_{j, \ell} n^{j}\left(\Delta_{n}(x)\right)^{j-\ell}, \\
\left|\tau_{j}^{(\ell)}(x, n)\right| \leq \text { const }_{j, \ell} n^{j}\left(\Delta_{n}(x)\right)^{j-\ell}
\end{array}
$$

for all $x \in[0,1]$. 
Proof Differentiate the formula (26) $\ell$ times to obtain

$$
T_{n j}^{(\ell)}(x)=\sum_{m \leq \min \{j, \ell\}}\left(\begin{array}{c}
\ell \\
m
\end{array}\right) \sum_{k=0}^{n}(-n)^{m} \frac{j !}{(j-m) !}(k-n x)^{j-m} p_{n k}^{(\ell-m)}(x) .
$$

By Lemma 17, each term is bounded by

$$
n^{m} \cdot n^{j-m} \cdot \operatorname{const}\left(\Delta_{n}(x)\right)^{(j-m)-(\ell-m)}=\operatorname{const} n^{j}\left(\Delta_{n}(x)\right)^{j-\ell},
$$

which proves the estimate (33). To get the analogous estimate for derivatives of $\tau_{j}=$ $\tau_{r j}$, we run an inductive argument. Differentiating the formula (29) $\ell$ times, we get

$$
\tau_{j}^{(\ell)}(x, n)=-\sum_{m \leq \ell}\left(\begin{array}{c}
\ell \\
m
\end{array}\right) \sum_{s=2}^{j} \frac{1}{s !} T_{n s}^{(m)}(x) \tau_{j-s}^{(\ell-m)}(x, n) .
$$

Applying the inductive assumption on the derivatives $\tau_{j-s}^{(\ell-m)}(x, n)$ and the already proven bound (33) on $T_{n s}^{(m)}(x)$, we obtain the estimate

$$
\text { const }_{j, \ell} n^{s}\left(\Delta_{n}(x)\right)^{s-m} n^{j-s}\left(\Delta_{n}(x)\right)^{j-s-\ell+m}=\text { const }_{j, \ell} n^{j}\left(\Delta_{n}(x)\right)^{j-\ell}
$$

on each term in the sum (35), and therefore on the function $\left|\tau_{j}^{(\ell)}(x, n)\right|$ as well, proving (34).

Next, we will show that the derivatives of the polynomials $Q_{n, r} f$ approximate the corresponding derivatives of $f$ sufficiently well. This is known as simultaneous approximation. Here is the precise result.

Lemma 19 Let $f \in C^{\alpha}[0,1]$, and let $r:=\lceil\alpha\rceil-1$. Then, for any $j=0, \ldots, r$,

$$
\left|\left(\left(I-Q_{n, r}\right) f\right)^{(j)}(x)\right| \leq C_{19}\|f\|_{C^{\alpha}}\left(\Delta_{n}(x)\right)^{\alpha-j} \quad \text { for all } x \in[0,1],
$$

where the constant $C_{19}$ is independent of $f$ and $n$.

Proof The case $j=0$ of this lemma is contained in formula (22) of [3]. To prove the result for all $j$, we use the fact that the Lorentz operator $Q_{n, r}$ reproduces polynomials of degree at most $r$.

Our goal is to show that the $j$ th derivative of the difference between $Q_{n, r} f$ and $f$ at any point $x$ is bounded by a constant multiple of $\|f\|_{C^{\alpha}}\left(\Delta_{n}(x)\right)^{\alpha-j}$ regardless of $x$. Since $Q_{n, r}$ reproduces polynomials of degree $r$ (by Lemma 14), we can subtract from $f$ its Taylor polynomial of degree $r$ centered at $x$ without changing the difference $\left(Q_{n, r} f-f\right)^{(j)}(x)$. Thus, without loss of generality we can assume that the value of 
$f$ and its derivatives up to order $r$ are zero at $x$. Now, recall that

$$
\begin{aligned}
\left(Q_{n, r} f-f\right)^{(j)}(x)= & \left(\sum_{k=0}^{n}\left(f\left(\frac{k}{n}\right)-f(x)\right) p_{n k}(x)\right. \\
& \left.+\sum_{i=2}^{r} \sum_{k=0}^{n} f^{(i)}\left(\frac{k}{n}\right) \frac{1}{n^{i}} \tau_{i}(x, n) p_{n k}(x)\right)^{(j)} .
\end{aligned}
$$

Differentiating these sums $j$ times, we will obtain terms of two kinds. Terms of the first kind are obtained from differentiating the first sum; they have the form

$$
\sum_{k=0}^{n}\left(f\left(\frac{k}{n}\right)-f(x)\right)^{(\ell)} p_{n k}^{(j-\ell)}(x)
$$

for some $\ell$ between 0 and $j$. Each of these sums can be bounded as follows, using Lemma 17:

$$
\begin{aligned}
\left|\sum_{k=0}^{n}\left(f\left(\frac{k}{n}\right)-f(x)\right)^{(\ell)} p_{n k}^{(j-\ell)}(x)\right| & \leq \sum_{k=0}^{n}\|f\|_{C^{\alpha}}\left|\frac{k}{n}-x\right|^{\alpha-\ell}\left|p_{n k}^{(j-\ell)}(x)\right| \\
& \leq \text { const }\|f\|_{C^{\alpha}}\left(\Delta_{n}(x)\right)^{(\alpha-\ell)-(j-\ell)} \\
& =\text { const }\|f\|_{C^{\alpha}}\left(\Delta_{n}(x)\right)^{\alpha-j} .
\end{aligned}
$$

Terms of the second kind are obtained by differentiating any of the other sums for $i=2, \ldots, r$ and have the form

$$
\sum_{k=0}^{n} f^{(i)}\left(\frac{k}{n}\right) \frac{1}{n^{i}} \tau_{i}^{(\ell)}(x, n) p_{n k}^{(j-\ell)}(x)
$$

for some $\ell$ between 0 and $j$. Taking into account that the derivatives of $f$ up to order $r$ vanish at $x$, each of these sums can be bounded by

$$
\begin{aligned}
& \left|\sum_{k=0}^{n} f^{(i)}\left(\frac{k}{n}\right) \frac{1}{n^{i}} \tau_{i}^{(\ell)}(x, n) p_{n k}^{(j-\ell)}(x)\right| \\
& \quad \leq \sum_{k=0}^{n}\|f\|_{C^{\alpha}}\left|\frac{k}{n}-x\right|^{\alpha-i} \frac{1}{n^{i}}\left|\tau_{i}^{(\ell)}(x, n)\right|\left|p_{n k}^{(j-\ell)}(x)\right| .
\end{aligned}
$$

Invoking the bound (34) from Lemma 18 on the terms $\left|\tau_{i}^{(l)}(x, n)\right|$, we conclude that the total is bounded by

$$
\text { const }\|f\|_{C^{\alpha}} \frac{1}{n^{i}} n^{i}\left(\Delta_{n}(x)\right)^{i-\ell} \sum_{k=0}^{n}\left|\frac{k}{n}-x\right|^{\alpha-i}\left|p_{n k}^{(j-\ell)}(x)\right| \text {. }
$$


The last sum, in turn, is estimated according to Lemma 17 to produce the final bound

$$
\text { const }\|f\|_{C^{\alpha}}\left(\Delta_{n}(x)\right)^{i-\ell} \cdot \Delta_{n}^{\alpha-i-j+\ell}(x)=\text { const }\|f\|_{C^{\alpha}}\left(\Delta_{n}(x)\right)^{\alpha-j} .
$$

This completes the proof.

Lemma 20 Let $f \in C^{\alpha}[0,1]$, and let $r:=\lceil\alpha\rceil-1$. Then, for all $x \in[0,1]$,

$$
\left|\left(Q_{n, r} f\right)^{(r+1)}(x)\right| \leq C_{20}\left(\Delta_{n}(x)\right)^{\alpha-r-1}\|f\|_{C^{\alpha}},
$$

with the constant $C_{20}$ independent of $f$ and $n$.

Proof First, we may assume without loss of generality that $f$ vanishes to order $r$ at $x$, since polynomials of degree at most $r$ are reproduced by $Q_{n, r}$ (Lemma 14) and then annihilated by taking the derivative of order $r+1$, as well as by taking the $r$ th derivative followed by a difference at two points $x$ and $y$. The assumption made above implies that, for all $i \leq r$,

$$
\left|f^{(i)}\left(\frac{k}{n}\right)\right| \leq \text { const }\|f\|_{C^{\alpha}}\left|\frac{k}{n}-x\right|^{\alpha-i} .
$$

By direct differentiation of (28),

$$
\left(Q_{n, r} f\right)^{(r+1)}(x)=\sum_{i=0}^{r} \sum_{\ell=0}^{r+1}\left(\begin{array}{c}
r+1 \\
\ell
\end{array}\right) \sum_{k=0}^{n} f^{(i)}\left(\frac{k}{n}\right) \frac{1}{n^{i}} \tau_{i}^{(\ell)}(x, n) p_{n k}^{(r+1-\ell)}(x) .
$$

Fix $i \in[0, r]$ and $\ell \in[0, r+1]$. The summand corresponding to $i$ and $\ell$ in (37) can be bounded by

$$
\begin{aligned}
& \left|\sum_{k=0}^{n} f^{(i)}\left(\frac{k}{n}\right) \frac{1}{n^{i}} \tau_{i}^{(\ell)}(x, n) p_{n k}^{(r+1-\ell)}(x)\right| \\
& \quad \leq \sum_{k=0}^{n}\|f\|_{C^{\alpha}}\left|\frac{k}{n}-x\right|^{\alpha-i} \frac{1}{n^{i}}\left|\tau_{i}^{(\ell)}(x, n)\right|\left|p_{n k}^{(r+1-\ell)}(x)\right| .
\end{aligned}
$$

Invoking Lemma 15 , we note that the terms $\frac{1}{n^{i}}\left|\tau_{i}^{(\ell)}(x, n)\right|$ are bounded by a constant multiple of $\left(\Delta_{n}(x)\right)^{i-\ell}$; therefore (38) is bounded by

$$
\text { const }\|f\|_{C^{\alpha}}\left(\Delta_{n}(x)\right)^{i-\ell}\left(\Delta_{n}(x)\right)^{\alpha-i-r-1+\ell}=\mathrm{const}\|f\|_{C^{\alpha}}\left(\Delta_{n}(x)\right)^{\alpha-r-1} .
$$

This proves (36).

Lemma 21 Let $f \in C^{\alpha}[0,1]$, and let $r:=\lceil\alpha\rceil-1$. Then, for any $x \in[0,1]$,

$$
\left\|Q_{n, r} f\right\|_{C^{\alpha}} \leq C_{21}\|f\|_{C^{\alpha}}
$$

with the constant $C_{21}$ independent of $f$ and $n$. 
Proof To establish the bound (39), we need to estimate the expression

$$
\left|\left(Q_{n, r} f\right)^{(r)}(x)-\left(Q_{n, r} f\right)^{(r)}(y)\right|
$$

for two points $x$ and $y$ in $[0,1]$. Without loss of generality, we may assume that $\Delta_{n}(x) \geq \Delta_{n}(y)$. Consider two cases.

Case 1. If $|x-y| \geq \Delta_{n}(x)$, then we estimate (40) using the triangle inequality and the bound

$$
\left|\left(Q_{n, r} f-f\right)^{(r)}(x)\right| \leq \operatorname{const}\left(\Delta_{n}(x)\right)^{\alpha-r}\|f\|_{C^{\alpha}}
$$

from Lemma 19 on each of the two terms, $\left(Q_{n} f-f\right)^{(r)}(x)$ and $\left(Q_{n} f-f\right)^{(r)}(y)$. Altogether, this bounds (40) from above by

$$
\text { const }\|f\|_{C^{\alpha}}\left(\Delta_{n}(x)\right)^{r-\alpha} \leq \text { const }\|f\|_{C^{\alpha}}|x-y|^{r-\alpha} \text {. }
$$

Case 2. If $|x-y| \leq \Delta_{n}(x)$, then $|x-y| \leq\left(\Delta_{n}(x)\right)^{r+1-\alpha}|x-y|^{\alpha-r}$, so for some $\xi$ between $x$ and $y$,

$$
\begin{aligned}
& \left|\left(Q_{n, r} f\right)^{(r)}(x)-\left(Q_{n, r} f\right)^{(r)}(y)\right| \\
& \quad=\left(Q_{n, r} f\right)^{(r+1)}(\xi) \cdot|x-y| \\
& \quad \leq \operatorname{const}\left(\Delta_{n}(\xi)\right)^{\alpha-r-1}\|f\|_{C^{\alpha}} \cdot\left(\Delta_{n}(x)\right)^{r+1-\alpha}|x-y|^{\alpha-r} .
\end{aligned}
$$

Lemma 12 implies that $\Delta_{n}(x) \leq 4 \Delta_{n}(\xi)$, and inserting this bound in (41) establishes (39).

Lemma 22 Suppose that $f:[0,1] \rightarrow \mathbb{R}$ satisfies $\left|f^{(r+1)}(x)\right| \leq\left(\Delta_{n}(x)\right)^{-\beta}$ for some $\beta \in[0,1]$ and all $x \in[0,1]$. Then, for all $x \in[0,1]$, we have

$$
\left|\left(Q_{n, r} f\right)^{(r+1)}(x)\right| \leq C_{22}\left(\Delta_{n}(x)\right)^{-\beta},
$$

with $C_{22}=C_{22}(r, \beta)$ a constant independent of $f$ and $n$.

Proof To prove (42), we may assume as in the preceding theorem that $f$ vanishes to order $r$ at $x$. This implies that for all $i \leq r$ and $z \neq x$ in $[0,1]$, there exists $\xi$ between $x$ and $z$ such that

$$
\frac{\left|f^{(i)}(z)\right|}{|z-x|^{r+1-i}} \leq\left|f^{(r+1)}(\xi)\right| \leq\left(\Delta_{n}(\xi)\right)^{-\beta} \leq 2\left(\Delta_{n}(x)\right)^{-\beta}\left(1+\frac{|x-z|}{\Delta_{n}(x)}\right),
$$

where the last step used (9) taken to the power $\beta$, and the inequality $|x-\xi| \leq|x-z|$.

Recall the expression (37) for $\left(Q_{n, r} f\right)^{(r+1)}(x)$. Fix $i \in[0, r]$ and $\ell \in[0, r+1]$. The summand

$$
\left|\sum_{k=0}^{n} f^{(i)}\left(\frac{k}{n}\right) \frac{1}{n^{i}} \tau_{i}^{(\ell)}(x, n) p_{n k}^{(r+1-\ell)}(x)\right|
$$


corresponding to $i$ and $\ell$ in (37) can be bounded using (43) and Lemma 15 by

$$
\text { const } \sum_{k=0}^{n}\left|\frac{k}{n}-x\right|^{r+1-i}\left(\Delta_{n}(x)\right)^{-\beta}\left(1+\frac{\left|\frac{k}{n}-x\right|}{\Delta_{n}(x)}\right)\left(\Delta_{n}(x)\right)^{i-\ell}\left|p_{n k}^{(r+1-\ell)}(x)\right| .
$$

Invoking Lemma 17 twice, we conclude that (44) is bounded by

$$
\operatorname{const}\left(\Delta_{n}(x)\right)^{-\beta}\left(\Delta_{n}(x)\right)^{i-\ell}\left(\left(\Delta_{n}(x)\right)^{\ell-i}+\frac{\left(\Delta_{n}(x)\right)^{\ell-i+1}}{\Delta_{n}(x)}\right) \leq \operatorname{const}\left(\Delta_{n}(x)\right)^{-\beta} \text {. }
$$

This proves the lemma.

\section{The Iterative Construction}

The goal of this section is to prove the sufficiency part of Theorem 8 . This will be achieved via an iterative construction of the approximants $f_{n}$ that are subsequently adjusted downward and upward to produce the approximants $g_{n}$ and $h_{n}$ satisfying the required consistency conditions listed in Result 3 in the introduction. We begin by analyzing the behaviour of the degree $n+r$ Bernstein coefficients of $Q_{n, r} f$.

Lemma 23 For every $\epsilon>0$, there exists $n_{0}$ such that for $n \geq n_{0}$, the degree $n+r$ Bernstein coefficients of $Q_{n, r} f$ are between $\min _{[0,1]} f-\epsilon$ and $\max _{[0,1]} f+\epsilon$.

Proof Recall that

$$
Q_{n, r} f=\sum_{k=0}^{n}\left(\sum_{j=0}^{r} \frac{f^{(j)}\left(\frac{k}{n}\right)}{n^{j}} \tau_{j}(x, n)\right) p_{n, k}(x) .
$$

The $i$ th Bernstein coefficient of $\tau_{j}(x, n) / n^{j} \in \mathcal{B}_{j}$ is bounded by $C_{j}^{\sharp} \min \left(n^{-i}, n^{i-j}\right)$ by Corollary 16. This implies that the Bernstein coefficients of $f^{(j)}\left(\frac{k}{n}\right) \tau_{j}(x, n) / n^{j}$ for $j \geq 1$ do not exceed $\frac{\text { const }}{n} \max _{1 \leq j \leq r}\left\|f^{(j)}\right\|_{\infty}$. Since the Bernstein coefficients of $\sum_{k=0}^{n} f\left(\frac{k}{n}\right) p_{n, k}(x)$ are between $\min _{[0,1]} f$ and $\max _{[0,1]} f$, we conclude that the Bernstein coefficients of $Q_{n, r} f \in \mathcal{B}_{n+r}$ are between $\min _{[0,1]} f-\frac{\text { const }}{n} \max _{1 \leq j \leq r}\left\|f^{(j)}\right\|_{\infty}$ and $\max _{[0,1]} f+\frac{\text { const }}{n} \max _{1 \leq j \leq r}\left\|f^{(j)}\right\|_{\infty}$, from which Lemma 23 follows immediately.

Lemma 24 Let $r:=\lceil\alpha\rceil-1$. If $f^{(j)}(x) \leq\left(\Delta_{n}(x)\right)^{\alpha-j}(j=0, \ldots, r)$ for all $x \in$ $[0,1]$, then the degree $n+r$ Bernstein coefficients of $Q_{n, r} f$ are dominated by those of

$$
C_{24}[x+(1-x)]^{r}\left[\sum_{k=0}^{n}\left(\Delta_{n}\left(\frac{k}{n}\right)\right)^{\alpha} p_{n, k}(x)\right],
$$

where $C_{24}$ does not depend on $n$ and $f$. 
Proof This lemma is a bit trickier. Separating the contributions given by different $j=0, \ldots, r$, we see that it would suffice to bound the coefficients of

$$
\left[\sum_{k=0}^{n}\left(\Delta_{n}\left(\frac{k}{n}\right)\right)^{\alpha-j} p_{n, k}(x)\right] \cdot \frac{\tau_{j}(x, n)}{n^{j}} \in \mathcal{B}_{n+j}
$$

by those of $\left[\sum_{k=0}^{n}\left(\Delta_{n}\left(\frac{k}{n}\right)\right)^{\alpha} p_{n, k}(x)\right][x+(1-x)]^{j}$ (possibly with some constant factor). Since both polynomials have symmetric coefficients as Bernstein polynomials in $\mathcal{B}_{n+r}$ and since we may assume without loss of generality that $n>3 r$, we see that it is enough to prove that

$$
\begin{aligned}
& \sum_{\substack{s+t=u \\
0 \leq s \leq u \\
0 \leq t \leq j}}\left(\begin{array}{l}
n \\
s
\end{array}\right)\left(\Delta_{n}\left(\frac{s}{n}\right)\right)^{\alpha-j} \min \left\{n^{-t}, n^{t-j}\right\} \leq C \sum_{\substack{s+t=u \\
0 \leq s \leq u \\
0 \leq t \leq j}}\left(\begin{array}{l}
n \\
s
\end{array}\right)\left(\Delta_{n}\left(\frac{s}{n}\right)\right)^{\alpha} \\
& \text { for } 0 \leq u \leq \frac{n+r}{2} \leq \frac{2 n}{3} .
\end{aligned}
$$

Note that $\Delta_{n}\left(\frac{s}{n}\right)$ is comparable to $\Delta_{n}\left(\frac{u}{n}\right)$ for $|s-u| \leq j \leq r$. This allows us to reduce the inequality to

$$
\sum_{\substack{s+t=u \\
0 \leq s \leq u \\
0 \leq t \leq j}}\left(\begin{array}{l}
n \\
s
\end{array}\right) \min \left\{n^{-t}, n^{t-j}\right\} \leq \mathrm{const}\left[\sum_{\substack{s+t=u \\
0 \leq s \leq u \\
0 \leq t \leq j}}\left(\begin{array}{l}
n \\
s
\end{array}\right)\right] \Delta_{n}^{j}\left(\frac{u}{n}\right) .
$$

We shall keep just one term $\left(\begin{array}{l}n \\ u\end{array}\right)\left(\Delta_{n}\left(\frac{u}{n}\right)\right)^{j}$ on the right and use the estimate $\left(\begin{array}{l}n \\ s\end{array}\right)=$ $\left(\begin{array}{c}n \\ u-t\end{array}\right) \leq 3^{t}\left(\frac{u}{n}\right)^{t}\left(\begin{array}{l}n \\ u\end{array}\right)$ valid for $u \leq \frac{2 n}{3}, t \geq 0$. Since $t \leq j \leq r$, we only need to show that

$$
\left(\frac{u}{n}\right)^{t} \min \left\{n^{-t}, n^{t-j}\right\}=\min \left\{\frac{u^{t}}{n^{2 t}}, \frac{u^{t}}{n^{j}}\right\} \leq C\left(\Delta_{n}\left(\frac{u}{n}\right)\right)^{j} .
$$

But $\left(\Delta_{n}\left(\frac{u}{n}\right)\right)^{j} \geq \frac{1}{n^{j}}$, which takes care of $u=0$ (with the only possible $t=0$ ), and if $1 \leq u \leq \frac{2 n}{3}$, we have $\Delta_{n}\left(\frac{u}{n}\right) \geq C \frac{u^{1 / 2}}{n}$, so it suffices to prove that $\min \left\{\frac{u^{t}}{n^{2 t}}, \frac{u^{t}}{n^{j}}\right\} \leq \frac{u^{j / 2}}{n^{j}}$ or equivalently, $\min \left\{\left(\frac{u}{n^{2}}\right)^{t-\frac{j}{2}}, u^{t-\frac{j}{2}}\right\} \leq 1$. But the first term is less than 1 for $t>\frac{j}{2}$, and the second term is not greater than 1 for $t \leq \frac{j}{2}$.

Iterative Construction of $f_{n} \quad$ Let $\alpha>0, \alpha \notin \mathbb{Z}$, hence $r=\lfloor\alpha\rfloor$. Assume that $f \in$ $C^{\alpha}[0,1]$ satisfies

$$
0<\min _{[0,1]} f \leq \max _{[0,1]} f<1 .
$$

Fix $n_{0} \in \mathbb{N}$ and $b=2^{s}$ to be chosen later. Denote $\Lambda:=\left\{b^{m} n_{0}: m \geq 0\right\}$, and define $f_{n}$ for $n \in \Lambda$ by

$$
\begin{aligned}
f_{n_{0}} & :=Q_{n_{0}, r} f, \\
f_{n} & :=f_{n / b}+Q_{n, r}\left(f-f_{n / b}\right) \text { for } n>n_{0} .
\end{aligned}
$$


Our task is to show that $f_{n} \rightarrow f$, that the Bernstein coefficients of $f_{n}$ are between $\delta$ and $1-\delta$ for some $\delta>0$, and that the Bernstein coefficients of $Q_{n, r}\left(f-f_{n / b}\right)$ are dominated (up to some constant factor) by those of

$$
[x+(1-x)]^{r}\left[\sum_{0 \leq k \leq n}\left(\Delta_{n}\left(\frac{k}{n}\right)\right)^{\alpha} p_{n, k}(x)\right] .
$$

We will do it in four steps.

Step 1. Estimate for $f_{n}^{(r+1)}$. We will show by induction that

$$
\left|f_{n}^{(r+1)}\right| \leq 2 C_{20}\|f\|_{C^{\alpha}}\left(\Delta_{n}\right)^{\alpha-r-1} \quad \text { on }[0,1],
$$

provided that $b$ is chosen large enough. By Lemma 20, the inequality (45) holds for $n=n_{0}$. Assume that it is true for $n / b$ in place of $n$. Write

$$
\left|f_{n}^{(r+1)}\right| \leq\left|f_{n / b}^{(r+1)}\right|+\left|\left(Q_{n, r} f_{n / b}\right)^{(r+1)}\right|+\left|\left(Q_{n, r} f\right)^{(r+1)}\right| .
$$

According to Lemma 20, the last term is bounded by $C_{20}\|f\|_{C^{\alpha}}\left(\Delta_{n}\right)^{\alpha-r-1}$. By the induction hypothesis,

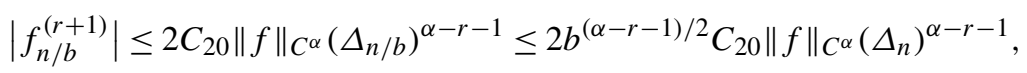

whence by Lemma 22 (with proper renormalization)

$$
\left|\left(Q_{n, r} f_{n / b}\right)^{(r+1)}\right| \leq 2 C_{22} b^{(\alpha-r-1) / 2} C_{20}\|f\|_{C^{\alpha}}\left(\Delta_{n}\right)^{\alpha-r-1} .
$$

If $b$ is chosen so large that $2\left(1+C_{22}\right) b^{(\alpha-r-1) / 2} \leq 1$, we can add these three estimates to get

$$
\left|f_{n}^{(r+1)}\right| \leq 2 C_{20}\|f\|_{C^{\alpha}}\left(\Delta_{n}\right)^{\alpha-r-1} .
$$

Moreover, we see that

$$
\left|\left[\left(I-Q_{n, r}\right) f_{n / b}\right]^{(r+1)}\right| \leq 2\left(1+C_{22}\right) b^{(\alpha-r-1) / 2} C_{20}\|f\|_{C^{\alpha}}\left(\Delta_{n}\right)^{\alpha-r-1} .
$$

Step 2. An estimate for $\left\|f_{n}\right\|_{C^{\alpha}}$. We will show that

$$
\left\|f_{n}\right\|_{C^{\alpha}} \leq 2 C_{21}\|f\|_{C^{\alpha}}, \quad \text { provided that } b \text { is large enough. }
$$

Again, we will argue by induction. Lemma 21 yields the base case $n=n_{0}$. Assume that the statement is true for $n / b$. Write

$$
f_{n}=\left(I-Q_{n, r}\right) f_{n / b}+Q_{n, r} f .
$$

Since $\left\|Q_{n, r} f\right\|_{C^{\alpha}} \leq C_{21}\|f\|_{C^{\alpha}}$ by Lemma 21 , it suffices to show that the $C^{\alpha}$-norm of the function

$$
\Psi:=\left(I-Q_{n, r}\right) f_{n / b}
$$

is bounded by $C_{21}\|f\|_{C^{\alpha}}$. 
We need to estimate $\left|\Psi^{(r)}(x)-\Psi^{(r)}(y)\right|$. Without loss of generality, we may assume that $\Delta_{n}(x) \geq \Delta_{n}(y)$. Choose a big positive constant $A$ and consider two cases:

Case 1. $|x-y| \geq A \Delta_{n}(x)$. Then

$$
\begin{aligned}
\left|\Psi^{(r)}(x)-\Psi^{(r)}(y)\right| & \leq\left|\Psi^{(r)}(x)\right|+\left|\Psi^{(r)}(y)\right| \\
& \leq 2 C_{19} C_{21}\|f\|_{C^{\alpha}}\left(\left(\Delta_{n}(x)\right)^{\alpha-r}+\left(\Delta_{n}(y)\right)^{\alpha-r}\right) \\
& \leq 4 C_{19} C_{21}\|f\|_{C^{\alpha}}\left(\Delta_{n}(x)\right)^{\alpha-r} \\
& \leq 4 C_{19} A^{-(\alpha-r)} C_{21}\|f\|_{C^{\alpha}}|x-y|^{\alpha-r},
\end{aligned}
$$

and we get the desired bound if $C_{19} A^{-(\alpha-r)} \leq 1$.

Case 2. $|x-y| \leq A \Delta_{n}(x)$. For this case, we will use the estimate

$$
\left|\Psi^{(r+1)}\right| \leq 2\left(1+C_{22}\right) b^{(\alpha-r-1) / 2} C_{20}\|f\|_{C^{\alpha}\left(\Delta_{n}\right)^{\alpha-r-1}}
$$

obtained in (46). Write

$$
\left|\Psi^{(r)}(x)-\Psi^{(r)}(y)\right|=\left|\Psi^{(r+1)}(\xi)\right||x-y|
$$

for some $\xi$ between $x$ and $y$. Now, by Lemma $12, \Delta_{n}(\xi) \geq[2(1+A)]^{-1} \Delta_{n}(x)$. Combining this with the above estimate for $\left|\Psi^{(r+1)}\right|$, we obtain

$$
\begin{aligned}
\left|\Psi^{(r+1)}(\xi)\right||x-y| \leq & 2\left(1+C_{22}\right) b^{(\alpha-r-1) / 2} C_{20}\|f\|_{C^{\alpha}}[2(1+A)]^{r+1-\alpha} \\
& \times A^{r+1-\alpha}\left[A \Delta_{n}(x)\right]^{\alpha-r-1}|x-y| \\
\leq & 2\left(1+C_{22}\right)[2 A(1+A)]^{r+1-\alpha} b^{(\alpha-r-1) / 2} C_{20}\|f\|_{C^{\alpha}|x-y|^{\alpha-r},}
\end{aligned}
$$

and we get the desired conclusion if

$$
2\left(1+C_{22}\right)[2 A(1+A)]^{r+1-\alpha} b^{(\alpha-r-1) / 2} \leq 1 .
$$

Step 3. An estimate for $\left(f-f_{n}\right)^{(j)}$. Since $f-f_{n}=\left(I-Q_{n, r}\right)\left(f-f_{n / b}\right)$ for $n \geq b n_{0}$ and we know that $\left\|f_{n / b}\right\|_{C^{\alpha}} \leq 2 C_{21}\|f\|_{C^{\alpha}}$, we can invoke Lemma 19 to conclude that

$$
\left|\left(f-f_{n}\right)^{(j)}\right| \leq C_{19}\left(1+2 C_{21}\right)\|f\|_{C^{\alpha}}\left(\Delta_{n}\right)^{\alpha-j} \quad \text { for } n \geq b n_{0} .
$$

The same, or an even better, estimate can be derived for $n=n_{0}$ from the representation $f-f_{n_{0}}=\left(I-Q_{n_{0}}\right) f$. In particular, we see that $f_{n} \rightarrow f$ uniformly in $[0,1]$.

Step 4. Estimates for Bernstein coefficients. It follows now from Lemma 24 and the result of the previous step that the degree $n+r$ Bernstein coefficients of $Q_{n, r}(f-$ $\left.f_{n / b}\right)$ are dominated by those of

$$
C_{24} C_{19}\left(1+2 C_{21}\right) b^{\alpha / 2}\|f\|_{C^{\alpha}}[x+(1-x)]^{r}\left[\sum_{k=0}^{n}\left(\Delta_{n}\right)^{\alpha}\left(\frac{k}{n}\right) p_{n, k}(x)\right]
$$


(here we used the inequality $\left(\Delta_{n / b}\right)^{j} \leq b^{j / 2}\left(\Delta_{n}\right)^{j} \leq b^{\alpha / 2}\left(\Delta_{n}\right)^{j}$ for $\left.0 \leq j \leq r\right)$. Since the latter coefficients are bounded by

$$
C_{24} C_{19}\left(1+2 C_{21}\right) b^{\alpha / 2}\|f\|_{C^{\alpha} n^{-\alpha / 2}},
$$

we see that the degree $n+r$ Bernstein coefficients of $f_{n}$ differ from those of $f_{n_{0}}$ at most by the factor

$$
\text { const } \sum_{n \in \Lambda, n>n_{0}} n^{-\alpha / 2} \leq C_{*} \cdot n_{0}^{-\alpha / 2} \text {. }
$$

Now, fix $\delta>0$ such that

$$
2 \delta<\min _{[0,1]} f \leq \max _{[0,1]} f<1-2 \delta
$$

and choose $n_{0}$ large enough so that $C_{*} \cdot n_{0}^{-\alpha / 2}<\delta$ and the degree $n+r$ coefficients of $Q_{n_{0}} f$ are between $2 \delta$ and $(1-2 \delta)$, which is possible by Lemma 23. Then the degree $n+r$ Bernstein coefficients of $f_{n}$ are between $\delta$ and $1-\delta$ for all $n \in \Lambda$ such that $n \geq n_{0}$.

Step 5. Construction of $g_{n}$ and $h_{n}$. Set

$$
\varphi_{n}(x):=\frac{\theta_{\alpha}}{n^{\alpha}}+\left[\frac{x(1-x)}{n}\right]^{\alpha / 2},
$$

where $\theta_{\alpha}$ will be specified later, and define

$$
g_{n}:=f_{n}-[x+(1-x)]^{r} D B_{n} \varphi_{n}, \quad h_{n}:=f_{n}+[x+(1-x)]^{r} D B_{n} \varphi_{n} .
$$

The constant $D$ here is to be chosen later. Clearly, the degree $n+r$ Bernstein coefficients of $h_{n}$ are greater than those of $g_{n}$. Also, since $\left|\varphi_{n}\right|<\delta D^{-1}$ for large $n$, we see that the Bernstein coefficients of $g_{n}$ are positive and those of $h_{n}$ are less than 1 for sufficiently large $n$. It remains to show that the Bernstein coefficients of $g_{n}$ "increase," those of $h_{n}$ "decrease," and that $g_{n}-h_{n}=O\left(\left(\Delta_{n}\right)^{\alpha}\right)$.

Lemma 25 The functions $\varphi_{n}$ defined in (47) satisfy

$$
B_{n} \varphi_{n} \leq \operatorname{const} \varphi_{n} \leq \operatorname{const}\left(\Delta_{n}\right)^{\alpha} .
$$

Consequently, $h_{n}-g_{n}=O\left(\left(\Delta_{n}\right)^{\alpha}\right)$.

Proof Since $\varphi_{n}$ is comparable to $\left(\Delta_{n}\right)^{\alpha}$, it suffices to show that

$$
\sum_{k=0}^{n}\left(\Delta_{n}\left(\frac{k}{n}\right)\right)^{\alpha} p_{n, k}(x) \leq C\left(\Delta_{n}(x)\right)^{\alpha}
$$

Recall that, by Lemma 12,

$$
\left(\Delta_{n}\left(\frac{k}{n}\right)\right)^{\alpha} \leq 2^{\alpha}\left(1+\frac{\left|x-\frac{k}{n}\right|}{\Delta_{n}(x)}\right)^{\alpha}\left(\Delta_{n}(x)\right)^{\alpha} \leq 2^{2 \alpha}\left(\left(\Delta_{n}(x)\right)^{\alpha}+\left|x-\frac{k}{n}\right|^{\alpha}\right) .
$$


Now, the first term yields the sum

$$
2^{2 \alpha}\left(\Delta_{n}(x)\right)^{\alpha} \sum_{k=0}^{n} p_{n, k}(x)=2^{2 \alpha}\left(\Delta_{n}(x)\right)^{\alpha},
$$

while the second one yields the sum

$$
2^{2 \alpha} \sum_{k=0}^{n}\left|x-\frac{k}{n}\right|^{\alpha} p_{n, k}(x) \leq \operatorname{const}\left(\Delta_{n}(x)\right)^{\alpha}
$$

due to Lemma 17. The desired bound $h_{n}-g_{n}=O\left(\left(\Delta_{n}\right)^{\alpha}\right)$ now follows from (48).

Next, we want to show that

$$
\left(B_{n} \varphi_{n}\right) \succeq_{2 n}(1+\gamma) B_{2 n} \varphi_{2 n},
$$

with some $\gamma>0$. To perform this comparison, we multiply the left-hand side by $[x+(1-x)]^{n}$ and expand. We thus see that this claim is equivalent to the system of inequalities

$$
\sum_{j=0}^{k} \frac{\left(\begin{array}{c}
n \\
j
\end{array}\right)\left(\begin{array}{c}
n \\
k-j
\end{array}\right)}{\left(\begin{array}{c}
2 n \\
k
\end{array}\right)} \varphi_{n}\left(\frac{j}{n}\right) \geq(1+\gamma) \varphi_{2 n}\left(\frac{k}{2 n}\right) \text { for } 0 \leq k \leq 2 n
$$

Denote the coefficients $\frac{\left(\begin{array}{c}n \\ j\end{array}\right)\left(\begin{array}{c}n \\ k-j\end{array}\right)}{\left(\begin{array}{c}2 n \\ k\end{array}\right)}$ by $\sigma_{k, j}$. Note that $\varphi_{n} \geq 2^{\alpha / 2} \varphi_{2 n}$, which immediately takes care of $k=0$ and $k=2 n$ with any $\gamma<2^{\alpha / 2}-1$. So, we will assume below that $1 \leq k \leq 2 n-1$.

Note that the function

$$
\Upsilon(x):=[x(1-x)]^{\alpha / 2}
$$

satisfies the inequality

$$
\frac{\Upsilon(x+t)+\Upsilon(x-t)}{2} \geq \Upsilon(x)\left[1-\frac{c_{\alpha}}{\min \{x, 1-x\}^{2}} t^{2}\right],
$$

for $0 \leq t \leq \min \{x, 1-x\}$, provided that $c_{\alpha}$ is large enough.

Indeed, when $0 \leq t \leq \frac{1}{2} \min \{x, 1-x\}$, (50) follows from the estimate $\left|\Upsilon^{\prime \prime}(\xi)\right| \leq$ const $\frac{\Upsilon(x)}{\min \{x, 1-x\}^{2}}$, valid for all $\xi \in[x-t, x+t]$, and when

$$
\frac{1}{2} \min \{x, 1-x\}<t \leq \min \{x, 1-x\}
$$

the inequality (50) is trivial, provided that $c_{\alpha} \geq 4$. Taking into account that $\sigma_{k, j}=$ $\sigma_{k, k-j}, \sum_{j} \sigma_{k, j}=1$ and that $\sigma_{k, j}=0$ if $\left|\frac{j}{n}-\frac{k}{2 n}\right|>\min \left\{\frac{k}{2 n}, 1-\frac{k}{2 n}\right\}$, we obtain

$$
\sum_{j} \sigma_{k, j} \Upsilon\left(\frac{j}{n}\right) \geq \Upsilon\left(\frac{k}{2 n}\right)\left[1-\frac{c_{\alpha} n^{2}}{\min \{k, 2 n-k\}^{2}} \sum_{j} \sigma_{k, j}\left(\frac{j}{n}-\frac{k}{2 n}\right)^{2}\right]
$$




$$
\begin{aligned}
& =\Upsilon\left(\frac{k}{2 n}\right)\left[1-\frac{c_{\alpha} k(2 n-k)}{\min \{k, 2 n-k\}^{2} 4(2 n-1)}\right] \\
& \geq \Upsilon\left(\frac{k}{2 n}\right)\left[1-\frac{c_{\alpha}}{4 \min \{k, 2 n-k\}}\right],
\end{aligned}
$$

because one of the factors $k$ and $2 n-k$ equals $\min \{k, 2 n-k\}$ and the other one does not exceed $2 n-1$. Thus

$$
\begin{aligned}
\sum_{j} \sigma_{k, j} \varphi_{n}\left(\frac{j}{n}\right) & =\frac{\theta_{\alpha}}{n^{\alpha}}+\frac{1}{n^{\alpha / 2}} \sum_{j} \sigma_{k, j} \Upsilon\left(\frac{j}{n}\right) \\
& \geq \frac{\theta_{\alpha}}{n^{\alpha}}+\frac{1}{n^{\alpha / 2}} \Upsilon\left(\frac{k}{2 n}\right)\left[1-\frac{c_{\alpha}}{4 \min (k, 2 n-k)}\right] .
\end{aligned}
$$

We have to compare that with

$$
(1+\gamma) \varphi_{2 n}\left(\frac{k}{2 n}\right)=\left[\frac{\theta_{\alpha}}{(2 n)^{\alpha}}+\frac{1}{(2 n)^{\alpha / 2}} \Upsilon\left(\frac{k}{2 n}\right)\right](1+\gamma) .
$$

Clearly, $\frac{\theta_{\alpha}}{n^{\alpha}}-\frac{(1+\gamma) \theta_{\alpha}}{(2 n)^{\alpha}} \geq\left(1-\frac{1+\gamma}{2^{\alpha}}\right) \frac{\theta_{\alpha}}{n^{\alpha}} \geq 0$ if $\gamma<2^{\alpha}-1$. Subtracting the second terms, we get

$$
\Upsilon\left(\frac{k}{2 n}\right) \frac{1}{n^{\alpha / 2}}\left[1-\frac{1+\gamma}{2^{\alpha / 2}}-\frac{c_{\alpha}}{4 \min (k, 2 n-k)}\right],
$$

which is nonnegative if $\gamma<2^{\alpha / 2}-1$ and if $k$ or $2 n-k$ is larger than some constant $K_{*}=K_{*}(\alpha, \gamma)$. But, for $\min (k, 2 n-k) \leq K_{*}$, we have $\Upsilon\left(\frac{k}{2 n}\right) \leq\left[\frac{K_{*}}{2 n}\right]^{\alpha / 2}$ and, thereby, the difference is (in absolute value) at most $\frac{c_{\alpha}}{4} \frac{K_{*}^{\alpha / 2}}{n^{\alpha}}$, which is dominated by $\left(1-\frac{1+\gamma}{2^{\alpha}}\right) \frac{\theta_{\alpha}}{n^{\alpha}}$, provided that $\theta_{\alpha}$ was chosen large enough. This proves (49).

An immediate corollary is that

$$
\left(B_{n / b} \varphi_{n / b}\right) \succeq_{n}(1+\gamma) B_{n} \varphi_{n}
$$

for every $n \in \Lambda \backslash\left\{n_{0}\right\}$. Thus, the Bernstein coefficients of

$$
[x+(1-x)]^{r}\left(B_{n / b} \varphi_{n / b}\right)[x+(1-x)]^{n-n / b}-[x+(1-x)]^{r} B_{n} \varphi_{n}
$$

are at least as large as those of $\gamma[x+(1-x)]^{r} B_{n} \varphi_{n}$. Since $\varphi_{n} \geq\left(\Delta_{n}\right)^{\alpha}$ (provided that $\theta_{\alpha} \geq 1$, of course), we see that the latter dominate the Bernstein coefficients of $Q_{n, r}\left(f-f_{n / b}\right)$ with some small constant. Choosing $D$ large enough, we turn this into true domination, which finishes the proof of "monotonicity" of the Bernstein coefficients of $g_{n}$ and $h_{n}$.

\section{Revisiting the Claim of Lorentz}

The goal of this section is to demonstrate that Lorentz' Claim 10 made in [3] is invalid. Our counterexample will be constructed in several steps. We begin with some elementary observations about Bernstein polynomials. 
Lemma 26 Let $\mathcal{B}_{n}^{+}[a, b]:=\left\{\sum_{k=0}^{n} c_{k}(x-a)^{k}(b-x)^{n-k}: c_{k} \geq 0\right\}$. Then

(a) $\mathcal{B}_{n}^{+}[a, b] \subset \mathcal{B}_{n+1}^{+}[a, b]$,

(b) $\mathcal{B}_{n}^{+}[a, b] \cdot \mathcal{B}_{m}^{+}[a, b] \subset \mathcal{B}_{n+m}^{+}[a, b]$,

(c) $\mathcal{B}_{n}^{+}[a, b] \subset \mathcal{B}_{n}^{+}[c, d]$ for every subinterval $[c, d]$ of the interval $[a, b]$,

(d) $\mathcal{B}_{n}^{+}[a, b]$ is a convex cone of functions.

Proof (a) Multiply by $1=\frac{1}{b-a}[(x-a)+(b-x)]$ and distribute.

(b) Multiply out.

(c) $x-a=(c-a)+(x-c) \in \mathcal{B}_{0}^{+}[c, d]+\mathcal{B}_{1}^{+}[c, d]=\mathcal{B}_{1}^{+}[c, d]$ and

$$
b-x=(b-d)+(d-x) \in \mathcal{B}_{0}^{+}[c, d]+\mathcal{B}_{1}^{+}[c, d]=\mathcal{B}_{1}^{+}[c, d] .
$$

Hence, $(x-a)^{k}(b-x)^{n-k} \in \mathcal{B}_{1}^{+}[c, d]^{n} \subset \mathcal{B}_{n}^{+}[c, d]$.

(d) Obvious.

Lemma 27 Suppose that $p$ is a polynomial of degree $n$ with real coefficients such that $p(0)>0$ and $p$ has no roots in the unit disc $\{|z| \leq 1\}$. Then $p \in \mathcal{B}_{n}^{+}[-1,1]$.

Proof We have $p(x)=\alpha \prod_{\beta}(x-\beta) \prod_{\gamma}(\gamma-x) \prod_{\lambda}(x-\lambda)(x-\bar{\lambda})$, where $\beta$ are negative roots, $\gamma$ are positive roots, $\lambda$ are complex roots with positive imaginary parts, and $\alpha>0$. Now

$$
x-\beta=(x+1)+(-\beta-1) \text { and } \quad-\beta-1>0 \text {. }
$$

Thus, $x-\beta \in \mathcal{B}_{1}^{+}[-1,1]$ for all $\beta$. Similarly, $\gamma-x \in \mathcal{B}_{1}^{+}[-1,1]$ for all $\gamma$. Now

$$
\begin{aligned}
& (x-\lambda)(x-\bar{\lambda})=x^{2}-2 \operatorname{Re}(\lambda x)+|\lambda|^{2} \text { is a convex combination of } \\
& (|\lambda|-x)^{2} \text { and }(x+|\lambda|)^{2} .
\end{aligned}
$$

Moreover, since $|\lambda|-x \in \mathcal{B}_{1}^{+}[-1,1]$ and $|\lambda|+x \in \mathcal{B}_{1}^{+}[-1,1]$, we infer that $(|\lambda|-x)^{2} \in \mathcal{B}_{2}^{+}[-1,1]$ and $(|\lambda|+x)^{2} \in \mathcal{B}_{2}^{+}[-1,1]$.

Lemma 28 The Taylor polynomial $P_{2 n}$ of degree $2 n$ of the function $\mathrm{e}^{-x^{2}}$ at 0 has no roots in the disc $\left\{|z| \leq \frac{\sqrt{n}}{\mathrm{e}}\right\}$.

Proof Let $|z| \leq \frac{\sqrt{n}}{\mathrm{e}}$. Then

$$
\begin{aligned}
\left|\mathrm{e}^{-z^{2}}-P_{2 n}(z)\right| & \leq \sum_{k>n} \frac{|z|^{2 k}}{k !} \leq \sum_{k>n}\left(\frac{\mathrm{e}|z|^{2}}{k}\right)^{k} \leq \sum_{k>n}\left(\frac{\mathrm{e}|z|^{2}}{n}\right)^{k} \leq \sum_{k>n} \mathrm{e}^{-k} \\
& <\mathrm{e}^{-n} \leq\left|\mathrm{e}^{-z^{2}}\right|
\end{aligned}
$$

and the result follows. 
In the sequel, we will make use of the inequality

$$
\left|\mathrm{e}^{-z^{2}}-P_{2 n}(z)\right|<\mathrm{e}^{-n} \quad \text { for }|z| \leq \frac{\sqrt{n}}{\mathrm{e}}
$$

obtained in the course of the last proof.

The following lemma is proved analytically, but the motivation of the construction is probabilistic. The Bernstein approximation $B_{n} f$ of a function $f$ can be viewed as the expectation of $f(X / n)$ where $X$ is a Binomial random variable with parameters $n$ and $x$. The Central limit theorem yields convergence of scaled Binomial variables to Gaussian variables, so the Bernstein approximation is close to the convolution of $f$ with a suitable Gaussian variable.

Lemma 29 Suppose that $v$ is a positive measure on $\mathbb{R}$ such that $g:=v * \mathrm{e}^{-n x^{2}}$ is bounded on the entire real line. Then there exists $p_{n} \in \mathcal{B}_{200 n}^{+}[-1,1]$ such that $\left\|g-p_{n}\right\|_{L^{\infty}[-1,1]} \leq 3 \mathrm{e}^{-n}\|g\|_{\infty}$.

Proof Note that $x \mapsto P_{200 n}(\sqrt{n}(x-t))$ has no roots in the disc $\left\{|z-t| \leq \frac{10}{\mathrm{e}}\right\}$. If $|t| \leq 2$, this disc contains the disc $\{|z| \leq 1\}$, so $P_{200 n}(\sqrt{n}(x-t)) \in \mathcal{B}_{200 n}^{+}[-1,1]$. Now set

$$
p_{n}=\left.v\right|_{[-2,2]} * P_{200 n}(\sqrt{n} \cdot) \in \mathcal{B}_{200 n}^{+}[-1,1] .
$$

For all $x \in[-1,1]$, we have

$$
\begin{aligned}
\left|g(x)-p_{n}(x)\right|= & \int_{2}^{\infty} \mathrm{e}^{-n(x-t)^{2}} \mathrm{~d} \nu(t)+\int_{-\infty}^{-2} \mathrm{e}^{-n(x-t)^{2}} \mathrm{~d} \nu(t) \\
& +\int_{-2}^{2}\left|\mathrm{e}^{-n(x-t)^{2}}-P_{200 n}(\sqrt{n}(x-t))\right| \mathrm{d} \nu(t) \\
=: & I_{1}+I_{2}+I_{3} .
\end{aligned}
$$

But $\left|\mathrm{e}^{-n(x-t)^{2}}-P_{200 n}(\sqrt{n}(x-t))\right| \leq \mathrm{e}^{-100 n}$ as long as $|x-t| \leq \frac{10}{\mathrm{e}}$. So $I_{3} \leq$ $\mathrm{e}^{-100 n} \int_{-2}^{2} \mathrm{~d} v(t)$. On the other hand, since

$$
\int_{-1}^{1} \mathrm{e}^{-n x^{2}} \mathrm{~d} x=\frac{1}{\sqrt{n}} \int_{-\sqrt{n}}^{\sqrt{n}} \mathrm{e}^{-x^{2}} \mathrm{~d} x \geq \frac{1}{2 \sqrt{n}}
$$

the definition of $g$ implies that

$$
\|g\|_{\infty} \cdot 6 \geq \int_{-3}^{3} g(x) \mathrm{d} x \geq \int_{-1}^{1} \mathrm{e}^{-n x^{2}} \mathrm{~d} x \int_{-2}^{2} \mathrm{~d} \nu(t)=\frac{1}{2 \sqrt{n}} \int_{-2}^{2} \mathrm{~d} \nu(t) .
$$

So $I_{3} \leq \mathrm{e}^{-100 n} 12 \sqrt{n}\|g\|_{\infty} \leq \mathrm{e}^{-95 n}\|g\|_{\infty}$. 
Now, since for every $y>0, z>1$, we have $\mathrm{e}^{-n(y+z)^{2}} \leq \mathrm{e}^{-n y^{2}} \mathrm{e}^{-n}$, we obtain

$$
\begin{aligned}
I_{1} & =\int_{2}^{\infty} \mathrm{e}^{-n(t-x)^{2}} \mathrm{~d} v(t)=\int_{2}^{\infty} \mathrm{e}^{-n((t-2)+(2-x))^{2}} \mathrm{~d} v(t) \\
& \leq \int_{2}^{\infty} \mathrm{e}^{-n} \cdot \mathrm{e}^{-n(t-2)^{2}} \mathrm{~d} v(t) \leq \mathrm{e}^{-n} g(2) \leq \mathrm{e}^{-n}\|g\|_{\infty},
\end{aligned}
$$

and, similarly, $I_{2} \leq \mathrm{e}^{-n}\|g\|_{\infty}$. Bringing these three estimates together, we arrive at the conclusion of the lemma.

Corollary 30 Let $E_{n}:=\left\{v * \mathrm{e}^{-n x^{2}}\right\}$, where the measure $v$ satisfies the assumption of Lemma 29. If $f: \mathbb{R} \rightarrow[0,1]$ can be approximated by functions $g_{n} \in E_{n}$ on the entire line with an error $O\left(n^{-\alpha / 2}\right)$, then $f$ can be approximated by $p_{n} \in \mathcal{B}_{n}^{+}[-1,1]$ with an error $O\left(n^{-\alpha / 2}\right)$ on $[-1,1]$.

Proof Obvious from Lemma 29.

Now fix $\alpha \in(0,1)$. Our next task will be to construct a function $f: \mathbb{R} \rightarrow[0,1]$ that is approximable by functions $g_{n} \in E_{\pi n}$ with an error $O\left(n^{-\alpha / 2}\right)$ but is not in the class $C^{\alpha}[-1 / 2,1 / 2]$. Note that $E_{\lambda} \subset E_{\lambda^{\prime}}$ whenever $\lambda<\lambda^{\prime}$, so it does not matter whether we consider only integer values or all real values of $n$ in our statement.

Fix $h \in(0,1)$ and $m \in \mathbb{N}$ and define

$$
f_{h, m}(x):=h \sum_{k \in \mathbb{Z}} \mathrm{e}^{-\pi m\left(x-\frac{k h}{\sqrt{m}}\right)^{2}}=h \sum_{k \in \mathbb{Z}} \mathrm{e}^{-\pi h^{2}\left(k-\frac{x \sqrt{m}}{h}\right)^{2}} .
$$

Recalling that the Fourier transform of the function $x \mapsto h \mathrm{e}^{-\pi h^{2} x^{2}}$ is $y \mapsto \mathrm{e}^{-\pi y^{2} / h^{2}}$ and using the Poisson summation formula

$$
\sum_{k \in \mathbb{Z}} F(k+x)=\sum_{\ell \in \mathbb{Z}} \widehat{F}(\ell) \mathrm{e}^{2 \pi i \ell x},
$$

we get

$$
f_{h, m}=\sum_{\ell \in \mathbb{Z}} \mathrm{e}^{-\pi \ell^{2} / h^{2}} \mathrm{e}^{-2 \pi i \ell x \sqrt{m} / h} .
$$

This representation immediately implies that $f_{h, m}$ attains its maximum at $x=0$, and that

$$
\begin{aligned}
\left|f_{h, m}-1\right| & \leq \sum_{\ell \in \mathbb{Z} \backslash\{0\}} \mathrm{e}^{-\pi \ell^{2} / h^{2}} \leq 2 \mathrm{e}^{-\pi / h^{2}}\left(1+\sum_{\ell \geq 3} \mathrm{e}^{-\pi \ell / h^{2}}\right) \\
& \leq 4 \mathrm{e}^{-\pi / h^{2}}, \\
f_{h, m}(0)-f_{h, m}\left(\frac{h}{2 \sqrt{m}}\right) & =2 \sum_{\ell \text { odd }} \mathrm{e}^{-\pi \ell^{2} / h^{2}}=2 \sum_{|\ell|=1} \mathrm{e}^{-\pi \ell^{2} / h^{2}}+\cdots \\
& \geq 4 \mathrm{e}^{-\pi / h^{2}} .
\end{aligned}
$$


Also note that $f_{h, m} \in E_{\pi m}$.

Now let $\Lambda$ denote the set $\left\{2^{j}: j=2,3,4, \ldots\right\}$. Choose $h_{m}$ so that

$$
\frac{\mathrm{e}^{-\pi / h_{m}^{2}}}{\left(\log _{2} m\right)^{2}}=\frac{1}{m^{\alpha / 2}}
$$

This choice makes sense for $m \geq m_{0}(\alpha) \geq 4$. Define $\Lambda^{\prime}:=\left\{m \in \Lambda: m \geq m_{0}(\alpha)\right\}$ and

$$
f:=\sum_{m \in \Lambda^{\prime}} \frac{1}{\left(\log _{2} m\right)^{2}} f_{h_{m}, m}
$$

For every $n \in \mathbb{N}$, let

$$
g_{n}:=\sum_{m \in \Lambda^{\prime}, m \leq n} \frac{1}{\left(\log _{2} m\right)^{2}} f_{h_{m}, m}+\sum_{m \in \Lambda^{\prime}, m>n} \frac{1}{\left(\log _{2} m\right)^{2}} \in E_{\pi n},
$$

since $g_{n}$ is a finite sum of elements of $E_{\pi m}$ with $m \leq n$ plus a constant. Now

$$
\begin{aligned}
\left|f-g_{n}\right| & \leq \sum_{m \in \Lambda^{\prime}, m>n} \frac{1}{\left(\log _{2} m\right)^{2}}\left\|f_{h_{m}, m}-1\right\|_{\infty} \leq \sum_{m \in \Lambda^{\prime}, m>n} \frac{1}{\left(\log _{2} m\right)^{2}} 4 \mathrm{e}^{-\pi / h_{m}^{2}} \\
& =4 \sum_{m \in \Lambda^{\prime}, m>n} m^{-\alpha / 2} \leq \mathrm{const} n^{-\alpha / 2} .
\end{aligned}
$$

On the other hand, for every $m \in \Lambda^{\prime}$, we have

$$
f(0)-f\left(\frac{h_{m}}{2 \sqrt{m}}\right) \geq\left(2 \sum_{\ell \text { odd }} \mathrm{e}^{-\pi \ell^{2} / h_{m}}\right)\left(\log _{2} m\right)^{-2}>4 \frac{\mathrm{e}^{-\pi / h_{m}^{2}}}{\left(\log _{2} m\right)^{2}}=4 m^{-\alpha / 2} .
$$

Thus,

$$
\|f\|_{C^{\alpha}[-1 / 2,1 / 2]} \geq \frac{4 m^{-\alpha / 2}}{\left(h_{m} /(2 \sqrt{m})\right)^{\alpha}}=16 h_{m}^{-\alpha} \rightarrow \infty \quad \text { as } m \rightarrow \infty,
$$

so $f$ is not in the class $C^{\alpha}[-1 / 2,1 / 2]$. So, we have obtained a function $f \notin$ $C^{\alpha}[-1 / 2,1 / 2]$ such that it can be approximated by polynomials $p_{n} \in \mathcal{B}_{n}^{+}[-1,1]$ at the rate $O\left(n^{-\alpha / 2}\right)$.

Consider the function $\tilde{f}(x):=f(x) \cdot x(1-x)$ and the polynomials $\widetilde{p}_{n}(x):=$ $p_{n}(x) \cdot x(1-x)$. The polynomials are in $\mathcal{B}_{n+2}^{+}[-1,1]$ and the function $\tilde{f}$ satisfies the condition

$$
\left|\tilde{f}(x)-\widetilde{p}_{n}(x)\right| \leq x(1-x) \text { const } n^{-\alpha / 2} \leq \mathrm{const}\left(\sqrt{\frac{x(1-x)}{n}}\right)^{\alpha} \leq \operatorname{const}\left(\Delta_{n}(x)\right)^{\alpha} .
$$

Claim 10 is thus disproved. 


\section{Further Questions and Remarks}

In this section we will make a few additional remarks on this and some related problems. We begin by discussing a conjectural characterization of simulation rates in case $\alpha$ is an integer. In that case, the classical problem of approximating a given function by polynomials of degree at most $n$ already has a somewhat different solution, as we now explain.

Definition 31 Let $\alpha \in \mathbb{N}$. A function $f$ is said to be in the smoothness class $C^{\alpha *}[0,1]$ if $f$ is $r:=\alpha-1$ times differentiable and the following condition holds:

The symmetric modulus of continuity of $f^{(r)}$

$$
\omega^{*}\left(f^{(r)}, h\right):=\sup _{t<h, x \in[t, 1-t]}\left|f^{(r)}(x+t)-2 f^{(r)}(x)+f^{(r)}(x-t)\right|
$$

is of order $O(h)$. In that case, we use the notation

$$
\|f\|_{C^{\alpha *}}:=\sup _{h} \frac{\omega^{*}\left(f^{(r)}, h\right)}{h} .
$$

Remark 32 The class $C^{\alpha *}[0,1]$ is also known as the generalized Lipschitz class, and, for $\alpha=1$, as the Zygmund class we already defined in Sect. 3 (also see [1, Chap. 2, Sect. 9]).

The characterization of the best polynomial approximation in case $\alpha \in \mathbb{N}$ is then given by the following result:

Result 33 (see, e.g., [1, Chap. 8, Theorem 6.3]) Let $\alpha \in \mathbb{N}$. There exists a sequence of polynomials $\left(p_{n}\right)$, where the degree of $p_{n}$ is at most $n$, satisfying

$$
\left|p_{n}(x)-f(x)\right|=O\left(\left(\Delta_{n}(x)\right)^{2 \alpha}\right) \quad \text { for all } x \in[0,1]
$$

if and only if $f \in C^{\alpha *}[0,1]$.

Motivated by this result on unrestricted polynomial approximation, we therefore conjecture a corresponding characterization of simulation rates.

Conjecture 34 Let $\alpha \in \mathbb{N}$. Let $f \in C^{\alpha *}[0,1]$ be a function bounded strictly between 0 and 1 . Then $f$ can be simulated at the rate $\left(\Delta_{n}(x)\right)^{\alpha}$ on $[0,1]$. Precisely, there exist polynomials $g_{n}$ and $f_{n}$ satisfying conditions (i)-(iv) of Result 3 and bound (7).

In Theorem 13 of Sect. 3, we have already verified the converse: if $f$ is simulable at the rate $\left(\Delta_{n}(x)\right)^{\alpha}$ on the interval $[0,1]$ where $\alpha \in \mathbb{N}$, then $f \in C^{\alpha *}[0,1]$.

Finally, we note that for any $\alpha>0$, it is natural to ask which functions $f$ can be simulated with a finite $\alpha$ moment, i.e., when is there a simulation algorithm for an $f(p)$-coin such that the number $N$ of tosses of $p$-coins and fair coins it uses satisfies

$$
\mathbf{E}_{p}\left(N^{\alpha}\right)\left(=\sum_{n=1}^{\infty} n^{\alpha} \mathbb{P}_{p}(N>n)\right)<\infty .
$$


We suspect that the precise criterion should involve the Besov smoothness of $f$, with proper attention to boundary effects; see, e.g., [1, pp. 54-57] for the definition and basic properties of Besov spaces.

Open Access This article is distributed under the terms of the Creative Commons Attribution Noncommercial License which permits any noncommercial use, distribution, and reproduction in any medium, provided the original author(s) and source are credited.

\section{References}

1. DeVore, R.A., Lorentz, G.G.: Constructive Approximation. Grundlehren der Mathematischen Wissenschaften [Fundamental Principles of Mathematical Sciences], vol. 303. Springer, Berlin (1993)

2. Keane, M.S., O'Brien, G.L.: A Bernoulli factory. ACM Trans. Model. Comput. Simul. 4(2), 213-219 (1994)

3. Lorentz, G.G.: The degree of approximation by polynomials with positive coefficients. Math. Ann. 151, 239-251 (1963)

4. Lorentz, G.G.: Bernstein Polynomials, 2nd edn. Chelsea Publishing, New York (1986)

5. Mossel, E., Peres, Y.: New coins from old: computing with unknown bias. Combinatorica 25(6), 707724 (2005). With an appendix by C. Hillar

6. Nacu, S., Peres, Y.: Fast simulation of new coins from old. Ann. Appl. Probab. 15(1A), 93-115 (2005)

7. Peres, Y.: Iterating von Neumann's procedure for extracting random bits. Ann. Stat. 20(1), 590-597 (1992)

8. Timan, A.F.: Theory of Approximation of Functions of a Real Variable. Dover, New York (1994). Translated from the Russian by J. Berry. Translation edited and with a preface by J. Cossar. Reprint of the 1963 English translation

9. von Neumann, J.: Collected Works. Vol. V: Design of Computers, Theory of Automata and Numerical Analysis. Pergamon Press, The Macmillan, New York (1963). General editor: A.H. Taub

10. Williams, D.: Probability with Martingales. Cambridge Mathematical Textbooks, Cambridge University Press, Cambridge (1991) 